\title{
Airborne hyperspectral observations of surface and cloud directional reflectivity using a commercial digital camera
}

\author{
A. Ehrlich ${ }^{1}$, E. Bierwirth ${ }^{1}$, M. Wendisch ${ }^{1}$, A. Herber ${ }^{2}$, and J.-F. Gayet ${ }^{3}$ \\ ${ }^{1}$ Leipzig Institute for Meteorology (LIM), University of Leipzig, Leipzig, Germany \\ ${ }^{2}$ Alfred Wegener Institute for Polar and Marine Research (AWI), Bremerhaven, Germany \\ ${ }^{3}$ Laboratoire de Météorologie Physique (LAMP), Université Blaise Pascal, Aubière Cedex, France
}

Correspondence to: A. Ehrlich (a.ehrlich@uni-leipzig.de)

Received: 27 June 2011 - Published in Atmos. Chem. Phys. Discuss.: 1 September 2011

Revised: 3 February 2012 - Accepted: 21 March 2012 - Published: 11 April 2012

\begin{abstract}
Spectral radiance measurements by a digital single-lens reflex camera were used to derive the directional reflectivity of clouds and different surfaces in the Arctic. The camera has been calibrated radiometrically and spectrally to provide accurate radiance measurements with high angular resolution. A comparison with spectral radiance measurements with the Spectral Modular Airborne Radiation measurement sysTem (SMART-Albedometer) showed an agreement within the uncertainties of both instruments $(6 \%$ for both). The directional reflectivity in terms of the hemispherical directional reflectance factor (HDRF) was obtained for sea ice, ice-free ocean and clouds. The sea ice, with an albedo of $\rho=0.96$ (at $530 \mathrm{~nm}$ wavelength), showed an almost isotropic HDRF, while sun glint was observed for the ocean HDRF $(\rho=0.12)$. For the cloud observations with $\rho=0.62$, the cloudbow - a backscatter feature typically for scattering by liquid water droplets - was covered by the camera. For measurements above heterogeneous stratocumulus clouds, the required number of images to obtain a mean HDRF that clearly exhibits the cloudbow has been estimated at about 50 images (10 min flight time). A representation of the HDRF as a function of the scattering angle only reduces the image number to about 10 ( 2 min flight time).

The measured cloud and ocean HDRF have been compared to radiative transfer simulations. The ocean HDRF simulated with the observed surface wind speed of $9 \mathrm{~m} \mathrm{~s}^{-1}$ agreed best with the measurements. For the cloud HDRF, the best agreement was obtained by a broad and weak cloudbow simulated with a cloud droplet effective radius of $R_{\text {eff }}=$ $4 \mu \mathrm{m}$. This value agrees with the particle sizes derived from in situ measurements and retrieved from the spectral radiance of the SMART-Albedometer.
\end{abstract}

\section{Introduction}

Surface reflectivity is a key parameter to estimate the Earth's atmosphere energy budget. As a lower boundary condition it is a parameter controlling the solar radiative transfer in the atmosphere. Considering the directional nature of radiometric quantities, such as radiance, the bidirectional reflectance distribution function (BRDF) fully describes the surface characteristics (e.g. Nicodemus et al., 1977; Schaepman-Strub et al., 2006). For the application of spaceborne instruments based on measurements of solar radiation, the BRDF is critical to retrieve aerosol or cloud properties. Hyer et al. (2011) found that correcting the surface albedo in the aerosol retrieval of the Moderate Resolution Imaging Spectroradiometer (MODIS) significantly reduces the variability of the bias between MODIS and ground based AOD measurements.

To estimate the impact of clouds on the Earth's energy budget from spaceborne measurements, the BRDF of clouds is required. Satellite instruments primarily measure spectral radiance and do not cover the entire hemisphere. However, the energy budget is calculated by hemispheric irradiance/top-of-atmosphere (TOA) albedo. To convert the satellite observations of reflectivity into TOA albedo, the cloud BRDF has to be known in terms of an angular distribution model (ADM, Loeb et al., 2000, 2005). From multiangular instruments such as the Clouds and the Earths Radiant Energy System (CERES, Loeb et al., 2005) and the Polarization and Directionality of the Earth's Reflectances instrument (POLDER, Loeb et al., 2000), empirical ADMs were derived from 24 and 5 months of observations, respectively. A different approach utilizing radiative transfer simulations was applied by Buriez et al. (2005) to measurements 
by POLDER. Plane-parallel radiative transfer calculations of the cloud BRDF for different cloud properties were used to convert the observations into TOA albedo.

However, for inhomogeneous clouds, plane-parallel radiative transfer calculations are not sufficient to simulate the angular reflectivity above clouds (e.g. Loeb and Davies, 1997; Varnai and Marshak, 2007). Analyzing observations of the Earth Radiation Budget Satellite (ERBS), Loeb and Davies (1997) found that plane-parallel simulations underestimate the reflectivity in the backscattering direction. Varnai and Marshak (2007) observed a bias in the cloud optical thickness retrieved by MODIS, which depends on the viewing angle of the sensor and cloud inhomogeneity. Both effects are significant for viewing angles of about $60^{\circ}$ and larger. Three-dimensional models may improve cloud BRDF simulations. However, given the diversity and complexity of clouds and the computational time of three-dimensional calculations, plane-parallel models are used for operative products, such as optical thickness and TOA albedo. These problems show that there is a need for measurements of the directional reflectivity above clouds.

Several ground-based and airborne retrieval techniques have been developed to derive the BRDF of different surfaces and clouds. While local ground-based measurements provide the BRDF of characteristic homogeneous surfaces (e.g. von Schönermark et al., 2004; Dumont et al., 2010), airborne data cover a larger measurement area and average over a mixture of different surface types, which is more suitable to the pixel size of spaceborne observations. However, for atmospheric measurements it has to be considered that the surface is illuminated by both the direct solar and the diffuse sky radiation. In this case, the measurements provide the hemispherical directional reflectance factor (HDRF) instead of BRDF. Based on radiative transfer calculations, the BRDF is derived afterwards by applying an atmospheric correction to the measured HDRF data.

State-of-the-art airborne BRDF instruments are mostly based on a scanning system measuring spectral radiance in different viewing angles. The Cloud Absorption Radiometer (CAR) presented by Gatebe et al. (2005) utilizes an optical system with a $1^{\circ}$ field of view. The mirror of the optical system is rotated at $100 \mathrm{r} \mathrm{min}^{-1}$. An entire BRDF measurement of the lower hemisphere is obtained within 2-3 min. BRDF measurements with CAR are reported above ocean, savanna, salt pans, snow and clouds (Gatebe et al., 2003; Lyapustin et al., 2010). A similar instrument including polarimetric data, the Research Scanning Polarimeter (RSP), is used by Litvinov et al. (2011) to validate BRDF models of vegetation and soil surfaces. The RSP employs a telescope with $0.8^{\circ}$ field of view and a double mirror with a scan rate of about $70 \mathrm{r} \mathrm{min}^{-1}$ to cover zenith angles of $\pm 60^{\circ}$ from the nadir direction.

Spaceborne multi-angular observations are obtained by instruments such as the Polarization and Directionality of the Earth's Reflectances instrument (POLDER, Descloitres et al., 1998) and the Multiangle Imaging SpectroRadiometer (MISR, Ovtchinnikov and Marchand, 2007). While POLDER provides a full image in $\pm 43^{\circ}$ along track and $\pm 51^{\circ}$ across track, MISR uses nine separate line cameras to cover nine different viewing angles. Using the airborne version of POLDER, Descloitres et al. (1998) compared the measured cloud HDRF (without atmospheric correction) to plane-parallel radiative transfer simulations, assuming spherical cloud particles. Differences ranged between $2 \%$ for liquid water clouds and $9 \%$ for ice clouds, which indicates that the scattering phase function of the cloud particles is essential for calculating HDRF. Assuming nonspherical ice crystals for the simulations, the differences are reduced to $2 \%$. With a similar approach, Ovtchinnikov and Marchand (2007) compared the radiance of different view angles measured by the airborne version of MISR and three-dimensional radiative transfer simulations. Differences appeared mainly in the nadir direction and are suggested to result from differences in the three-dimensional structure between observed and simulated clouds.

Here we present airborne measurements of HDRF using a commercial, single-lens reflex digital camera. Compared to scanning instruments, digital cameras instantly obtain a full scene of measurements without the need of high-precision movable components. The camera is easy to mount on an aircraft and relatively cheap. The high spatial resolution of the camera allows measurements with an angular resolution of about $0.1^{\circ}$. However, due to the imaging system including lens and sensor, a careful calibration of the camera is required to quantify the angular dependence of the camera sensitivity, which might be affected by dark noise, saturation, distortion, or polarization effects.

Such a type of camera is still rarely applied in atmospheric sciences, even though there is an increasing use in vegetation and soil monitoring (Lebourgeois et al., 2008). Only a few studies have used such camera measurements quantitatively. From radiance-calibrated conventional photographs, Cox and Munk (1954) derived a parametrization of ocean BRDF. Digital cameras were introduced in the last century for ground-based cloud-cover detection (e.g. Long et al., 2006; Schade et al., 2009). However, instead of calibrated radiance, Long et al. (2006) and Schade et al. (2009) used the radiance-uncalibrated signals of the camera sensor to detect clouds by analyzing the three spectral channels (red, green, blue; RGB) of the CCD (charged coupled device) sensor.

We analyze radiance-calibrated digital camera images obtained from airborne measurements performed during a campaign in the Arctic. They are introduced in Sect. 2. The processing of the digital camera images, including radiometric and spectral calibration, is shown in Sect. 3. HDRF measurements for different surfaces are presented in Sect. 4. The results for cloud and ocean HDRF are discussed and compared to radiative transfer simulations in Sect. 5. Section 6 presents the conclusions of this paper. 


\section{Instrumentation and measurements}

We report on data collected during the Solar Radiation and Phase discrimination of Arctic Clouds (SORPIC) campaign in May 2010. During SORPIC the Polar 5 aircraft, owned by the Alfred Wegener Institute for Polar and Marine Research (AWI), Bremerhaven, Germany, was deployed to investigate Arctic clouds with a set of remote sensing and in situ instruments. With the Polar 5 based in Longyearbyen on Svalbard $\left(78^{\circ} 13^{\prime} \mathrm{N}, 15^{\circ} 38^{\prime} \mathrm{E}\right)$, in total 13 flights were conducted covering the area of the Greenland Sea west of Svalbard.

The major purpose of the flights was to quantify the horizontal and vertical distribution of ice and liquid water in mixed-phase clouds by different independent approaches, including remote sensing and in situ measurements. The airborne instrumentation for remote sensing included the Spectral Modular Airborne Radiation measurement sysTem (SMART-Albedometer), the hyperspectral camera system AISA Eagle, the Airborne Mobile Aerosol Lidar (AMALi), and a commercial CANON EOS-1D Mark III digital camera. Additionally, an airborne sun photometer was operated to characterize aerosol properties. For in situ measurements, a Nevzorov probe, the Polar Nephelometer, a Cloud Particle Imager (CPI), and the Particle Measuring System (PMS) Forward Scattering Spectrometer Probe (FSSP-100) were installed on Polar 5. A detailed description of the instrumentation is given by Lampert et al. (2009) and Gayet et al. (2009). The SMART-Albedometer was described by Wendisch et al. (2001) and Ehrlich et al. (2008). For sea ice measurements, the electromagnetic-induction (EM) system EM-bird was operated in a towed sonde during the flights on 13 and 14 May 2010 (Haas et al., 2009).

To demonstrate the potential of HDRF measurements with the CANON camera, we present three selected cases of measurements above clouds, sea ice, and open water. For the cloud case, we focus on observations of pure liquid water clouds observed on 17 May 2010 south of Svalbard over icefree sea. A strong advection of warm air produced a persistent cloud layer in the lower boundary layer, with cloud top rising from $200 \mathrm{~m}$ in the south to $700 \mathrm{~m}$ in the north. Measurements above sea ice and open water were obtained during a flight with clear sky conditions on 14 May 2010. The sea ice was observed at about $82^{\circ} \mathrm{N}, 2^{\circ} \mathrm{W}$; the open water at about $79^{\circ} 20^{\prime} \mathrm{N}, 10^{\circ} \mathrm{E}$. In all cases, clear sky was reported above the aircraft.

\section{Digital camera}

\subsection{General characteristics}

The CANON EOS-1D Mark III is a digital single-lens reflex (DSLR) camera, which incorporates a CMOS (Complementary Metal Oxide Semiconductor) image sensor providing the three spectral channels (RGB). The advantage of the CMOS

\section{Interior View}
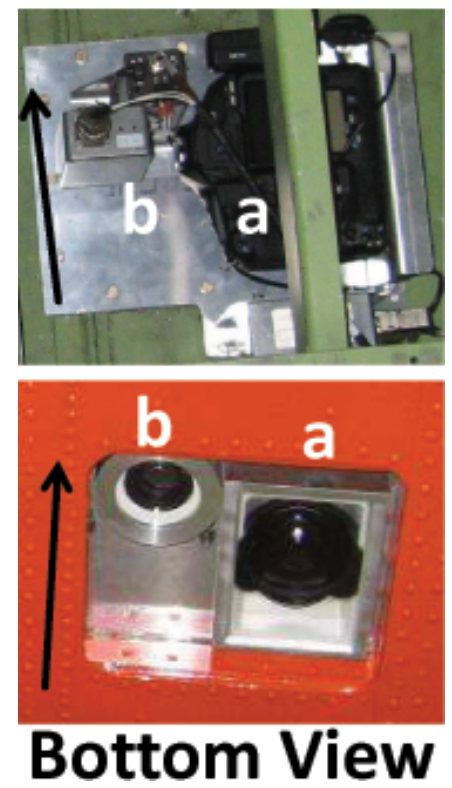

Fig. 1. Photographs of the installation of the CANON camera on board of Polar 5 (arrows indicate the flight direction). In both photographs (interior and bottom view) the CANON camera is labeled with (a) and the digital video camera with (b).

image sensor compared to CCD sensors is the possibility of using larger sensors with low power consumption. This allows pixels with larger surface area, which increases the dynamic range of the sensor. With the new sensor generation, the noise and dark current level of CMOS sensors have been reduced to typical values of CCD sensors (Kaufmann, 2010).

The CMOS sensor applied in the CANON EOS-1D Mark III has the Advanced Photo System APS-H format with a $28.1 \times 18.7 \mathrm{~mm}$ sensor area (crop factor of 1.3 ). The sensor has a $3908 \times 2600$ pixel grid and covers a total of about $10.2 \times 10^{6}$ pixels (10 megapixels).

To cover a large area, the camera was configured with the wide-angle lens Canon EF 14 mm f/2.8L II USM. Compared to a fisheye lens, this lens provides distortion-free images all the way across the frame. Thus, the camera field of view $\Theta$ is calculated from the lens focal length of $f=14 \mathrm{~mm}$ and the sensor chip size $d$ :

$\Theta=2 \cdot \arctan \left(\frac{d}{2 f}\right)$.

For the horizontal $(d=28.1 \mathrm{~mm})$ and vertical $(d=$ $18.7 \mathrm{~mm}$ ) direction, the angle of view is $\Theta=90.2^{\circ}$ and $\Theta=67.5^{\circ}$, respectively. The image diagonal has an angle of view of $\Theta=100.6^{\circ}$. The corresponding angular resolution of each pixel is about $0.025^{\circ}$.

The camera was installed on Polar 5 close to a low definition digital video camera, as shown in Fig. 1. To protect the camera lens from damage by stone chipping and rain water, a 
glass window was integrated in the aircraft frame in front of the lens. The camera was fixed to the aircraft frame, which made a correction to the aircraft attitude necessary. To guarantee the overlap of at least two subsequent images, the camera was aligned with its long image side along the aircraft axis.

To obtain the full dynamic range of the camera sensor chip, only raw data (RAW) were analyzed. Compared to the standard JPG format ( 8 bit), the RAW format provides 16 bit dynamic range. To read the camera manufacturer-specific RAW format (Canon RAW version 2, CR2), we employed the open source tool DCRAW (http://www.cybercom.net/ dcoffin/dcraw/). With DCRAW, the CR2 images were converted into portable pixmap format (PPM) files using the command:

$$
\text { dcraw -c -v -t } 0 \text {-o } 0 \text {-r } 11111 \text {-k } 0 \text {-S } 16384 \text {-4 }
$$

To avoid any manipulation of the original measurements, no white balance was applied by setting the multipliers of all channels to 1 . The darkness level was set to 0 and the saturation level to 16384 , respectively, with linear interpolation in between. Finally, the dark current of the images was determined in the laboratory for different camera settings and environmental conditions. Images without illumination were taken for temperatures between $10^{\circ} \mathrm{C}$ and $25^{\circ} \mathrm{C}$. All data were taken with the same exposure as used during the airborne measurements (1/2656s), which showed that the dark current does not exceed one digital unit. Thus the dark current is negligible, which agrees with Kaufmann (2010) who found that the dark current is no issue for exposure times below $33 \mathrm{~ms}$.

\subsection{Spectral calibration}

To compare the camera measurements with spectral measurements of the SMART-Albedometer and radiative transfer simulations, the spectral sensitivity of each RGB channel was determined in the laboratory. The camera was mounted in front of a grating monochromator (Zolix Omni- $\lambda 300$ ). A $200 \mathrm{~W}$ halogen lamp was used as radiation source. The spectral irradiance emitted by the lamp was determined by cross calibration of a $1000 \mathrm{~W}$ halogen lamp traceable to the National Institute of Standards and Technology (NIST) standard. Measurements with the camera were made between $300 \mathrm{~nm}$ and $700 \mathrm{~nm}$ wavelength for steps of $5 \mathrm{~nm}$. For the monochromator, a grating with a blaze wavelength of $500 \mathrm{~nm}$ and a groove density of $1200 \mathrm{~mm}^{-1}$ was chosen, providing a spectral resolution of $0.1 \mathrm{~nm}$. The wavelength accuracy of the monochromator is specified as $0.2 \mathrm{~nm}$. The bandwidth was set to $5 \mathrm{~nm}$, providing a sufficiently high radiance to be detected by the camera.

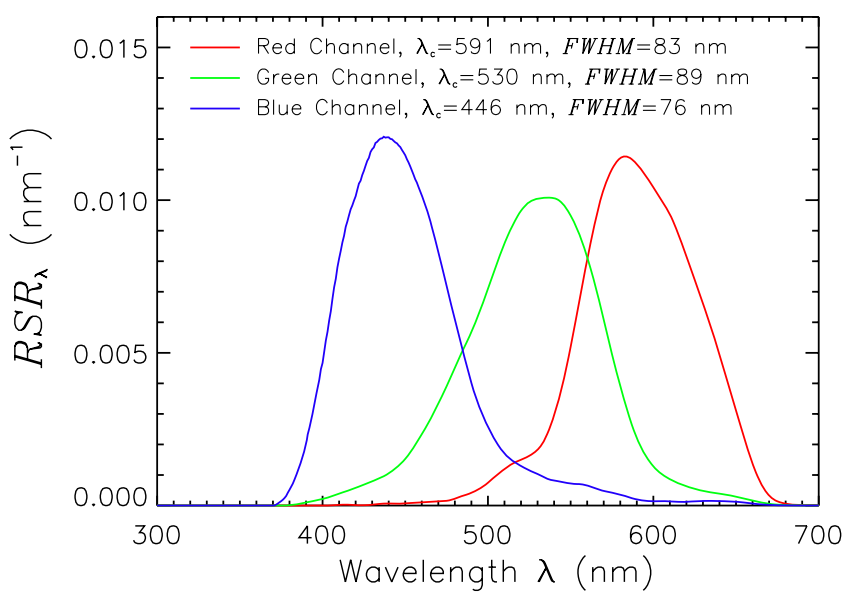

Fig. 2. Relative spectral response function $\operatorname{RSR}_{\lambda}$ of the three camera channels (red, green, blue). For each channel, the center wavelength (median value) $\lambda_{\mathrm{c}}$ and the FWHM are given.

The relative spectral response function $\mathrm{RSR}_{\lambda}$ is defined by the normalization:

$\int_{0}^{\infty} \operatorname{RSR}_{\lambda} \mathrm{d} \lambda=1$.

It is calculated from the measured camera signal $S_{\lambda}$, and the irradiance $F_{1, \lambda}$ emitted by the $200 \mathrm{~W}$ halogen lamp by:

$\operatorname{RSR}_{\lambda}=\frac{S(\lambda)}{F_{1, \lambda}} \cdot\left(\int_{0}^{\infty} \frac{S(\lambda)}{F_{1, \lambda}} \mathrm{d} \lambda\right)^{-1}$.

The $\operatorname{RSR}_{\lambda}$ function measured in the laboratory is shown in Fig. 2 for all three camera channels. The RSR ${ }_{\lambda}$ of all channels is non-Gaussian, with their full-width of half-maximum (FWHM) ranging between $76 \mathrm{~nm}$ for the blue channel and $89 \mathrm{~nm}$ for the green channel. The center wavelength $\lambda_{\mathrm{c}}$ of each channel (median value of $\mathrm{RSR}_{\lambda}$ ) was determined as $591 \mathrm{~nm}$ (red, channel 1), $530 \mathrm{~nm}$ (green, channel 2), and $446 \mathrm{~nm}$ (blue, channel 3).

\subsection{Radiometric calibration}

The exposure time of the camera, aperture (f-number), and film speed were fixed during the measurements. The settings with an exposure time of $1 / 2656 \mathrm{~s}$, an f-number of F/9.1, and a film speed of ISO-400 were chosen for cloud and sea ice observations with high reflectivities, but these settings worked as well for measurements above the open ocean. The short exposure time was chosen to avoid distortion due to the aircraft movement.

The radiometric calibration was obtained in the laboratory with the use of a NIST traceable radiance source (integrating sphere). The camera was mounted in the laboratory together with the protective glass window required for the aircraft installation (Fig. 1) in front of the aperture of the integrating 

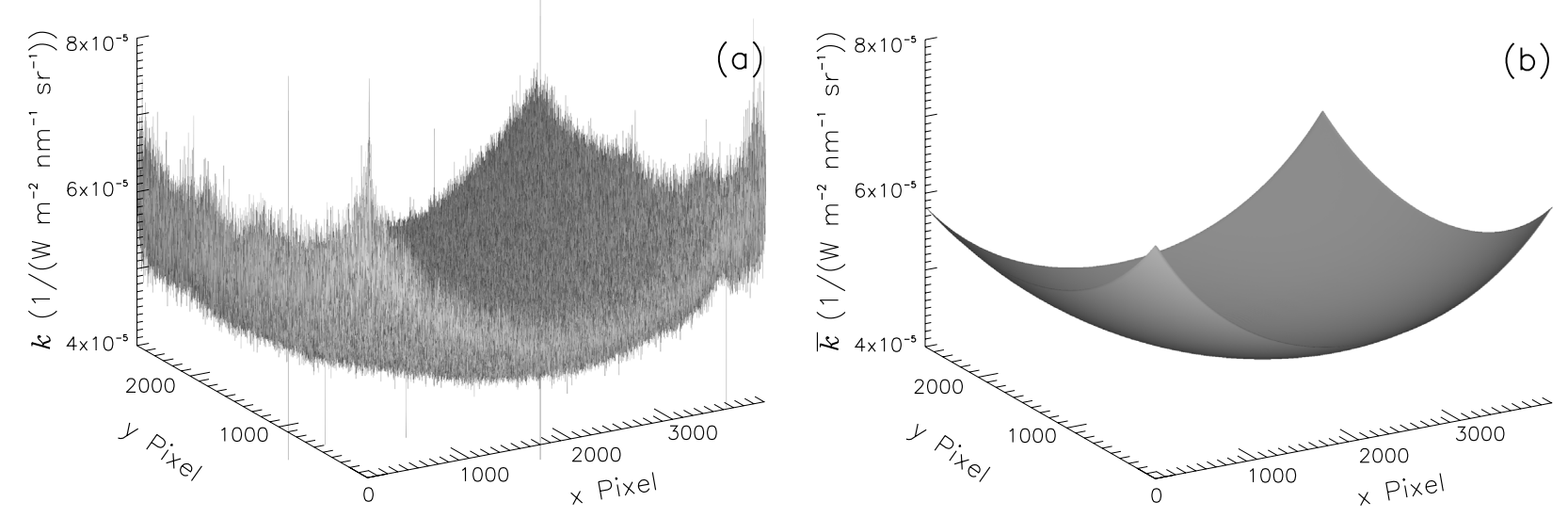

Fig. 3. Radiometric calibration coefficients $k$ and $\bar{k}$ of camera channel $1(\lambda=591 \mathrm{~nm})$. Panel (a) shows the noisy raw data $(k)$. In Panel $(\mathbf{b})$ a two-dimensional fit was applied to smooth the data $(\bar{k})$. The calibration is valid for an exposure time of 1/2656 s, an $\mathrm{f}-\mathrm{number}$ of $\mathrm{F} / 9.1$, and a film speed of ISO-400.

sphere at $5 \mathrm{~cm}$ and $15 \mathrm{~cm}$ distances. For both distances the exit port of the integrating sphere with $6 \mathrm{~cm}$ diameter did not cover the whole image. Therefore, a series of images was taken while the camera was moved horizontally and vertically. No differences between measurement at both distances were observed. Therefore, all images were merged into a single calibration.

The calibration coefficients $k_{\lambda}$ were calculated for each camera pixel $(x, y)$ and each camera channel using the camera signal $S_{\lambda, \mathrm{C}}(x, y)$ (digital counts) and the NIST traceable radiance emitted by the integrating sphere $I_{\lambda, \text { IS }}$ by:

$k_{\lambda}(x, y)=\frac{S_{\lambda, \mathrm{C}}(x, y)}{I_{\lambda, \mathrm{IS}}}$.

Figure 3 a shows the original calibration coefficients $k_{\lambda}$ of the merged images for channel $1(591 \mathrm{~nm})$. The plot indicates that the raw data of the camera is noisy. The noise is typical for CMOS image sensors and randomly distributed independent of the pixel position, as shown by laboratory tests (not shown here). Compared to channel 1, the noise of channel 3 $(446 \mathrm{~nm})$ is of similar magnitude, while channel $2(530 \mathrm{~nm})$ shows a reduced noise level. This is probably caused by the doubled number of channel 2 pixels of the Bayer filter used in the CMOS sensor. The data analysis is not seriously effected by the noise, as it is counterbalanced by the high number of pixels. To remove the noise in the calibration, a two-dimensional polynomial fit of 4th degree was applied to smooth the data. The final calibration coefficients $\overline{k_{\lambda}}$ used to process the data are shown in Fig. $3 \mathrm{~b}$ for channel 1. It shows that the sensitivity of the CMOS sensor is maximal in the center and decreases towards the edges of the sensor. The difference between maximum and minimum is about $40 \%$. This vignetting effect is well known for digital cameras (see Lebourgeois et al., 2008; Olsen et al., 2010). This pattern has been observed in all the three channels, indicating that the pattern results from lens effects.

Lebourgeois et al. (2008) corrected the vignetting effect by fitting a polynomial function onto an average image of about 500 images. This method does not work if the observed surface is a non-isotropic reflector itself, e.g. sea ice, clouds, or open water. For such surfaces, the vignetting effect will be superimposed by the BRDF of the surface. In this case the vignetting effect has to be eliminated by a radiometric calibration, as presented above for the CANON camera.

Polarized radiation (e.g. sun glint) might increase the uncertainty of the camera measurements if the camera lens acts like a polarization filter. The sensitivity to linear polarized radiation of different orientation was tested in the laboratory using a source of $100 \%$ linear polarized radiation. Differences between measurements of parallel and perpendicular polarized radiation were found to be negligible for the center of the images. Toward the edge of the image, this polarization effect slightly increased. Maximum effects were estimated to be $3 \%$. It has to be taken into account that for radiation, which is not $100 \%$ polarized, this effect will be reduced by the degree of polarization.

The sensitivity of the CMOS image sensor was additionally tested for linearity. The results (not shown here) agree with the study reported by Kaufmann (2010), who showed an almost perfect linear response of the CMOS image sensor to the intensity of the incoming radiation. Thus, the uncertainty in the radiometric calibration results mainly from the uncertainty given for the certified radiance source. For the camera setup used in this study, an uncertainty in the radiance measurements of about $7 \%$ was considered for each camera channel. 

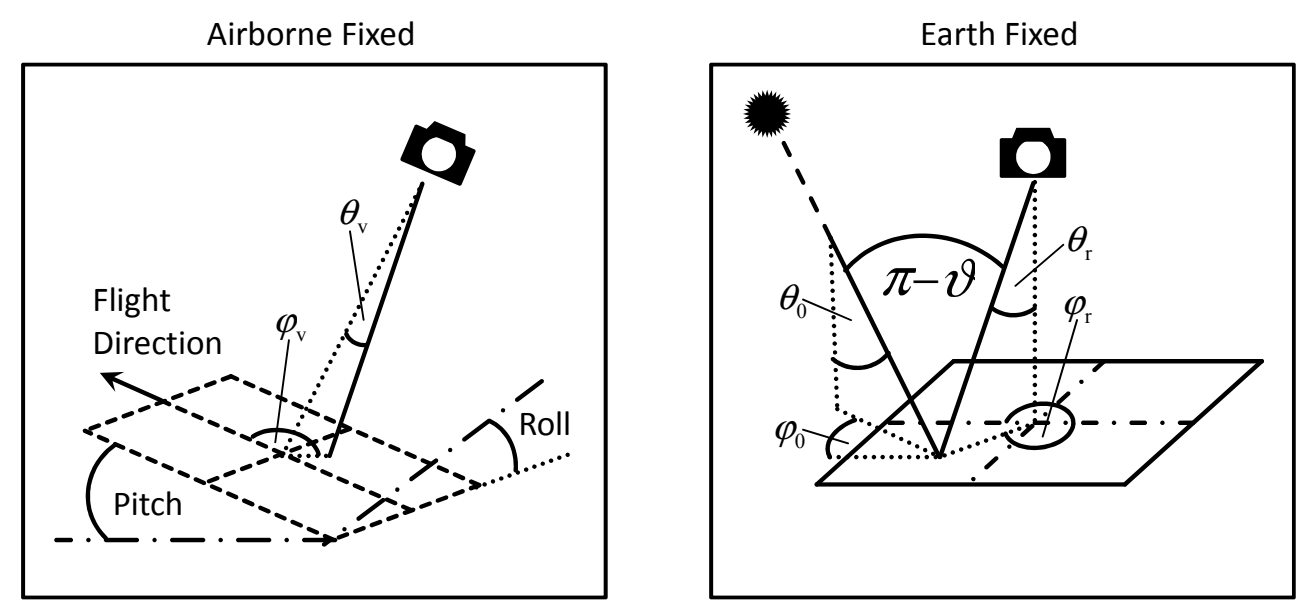

Fig. 4. Illustration of the airborne fixed (left, $\theta_{\mathrm{v}}, \phi_{\mathrm{V}}$ ) and Earth fixed coordinates (right, $\theta_{\mathrm{r}}, \varphi_{\mathrm{r}}$ ) of one single camera pixel. Additionally, the scattering angle $\vartheta$ is indicated with the position of the Sun defined by $\theta_{0}, \varphi_{0}$.

\subsection{Geometry}

As the camera is fixed to the aircraft frame, a correction for the aircraft attitude has to be applied before averaging different images. The definition of the coordinate systems is shown in Fig. 4, where the position of the Sun is defined by the solar zenith angle $\theta_{0}$ and the solar azimuth angle $\varphi_{0}$. The pixel coordinates are given by the viewing zenith angle $\theta_{\mathrm{v}}$ and the viewing azimuth angle $\varphi_{\mathrm{v}}$. The viewing zenith angle is derived from Eq. (1) by replacing the diameter of the sensor with the corresponding distance of each pixel to the center of the sensor. The viewing azimuth angle is defined clockwise, with $0^{\circ}$ showing into flight direction.

$\theta_{\mathrm{v}}$ and $\varphi_{\mathrm{v}}$ have been corrected for the aircraft roll and pitch angle. Therefore, Euler rotations of the pixel coordinates with roll and pitch angles were applied. The rotation of $\theta_{\mathrm{v}}$ and $\varphi_{\mathrm{v}}$ gives $\theta_{\mathrm{r}}$ and $\varphi_{\mathrm{r}}$, the zenith and azimuth angles of the reflected radiation in Earth fixed coordinates. Finally, the images have been rotated into the azimuthal direction of the Sun $\varphi_{0}$.

Assuming single scattering, the scattering angle $\vartheta$ of direct solar radiation has been calculated for each image pixel (e.g. Wendisch and Yang, 2012). $\vartheta$ is defined as the angle between the direction of the Sun $\left(\theta_{0}, \varphi_{0}\right)$ and the viewing direction $\left(\theta_{\mathrm{r}}\right.$, $\left.\varphi_{\mathrm{r}}\right)$, and is calculated by:

$$
\begin{array}{r}
\vartheta=180^{\circ}-\arccos \left(-\sin \theta_{0} \cdot \cos \varphi_{0} \cdot \sin \theta_{\mathrm{r}} \cdot \cos \varphi_{\mathrm{r}}\right. \\
-\sin \theta_{0} \cdot \sin \varphi_{0} \cdot \sin \theta_{\mathrm{r}} \cdot \sin \varphi_{\mathrm{r}} \\
\left.+\cos \theta_{0} \cdot \cos \theta_{\mathrm{r}}\right) .
\end{array}
$$

\subsection{Hemispherical-directional reflectance factor HDRF}

The reflectivity of surfaces is generally described by the bidirectional reflectance distribution function BRDF (Nicodemus et al., 1977; Schaepman-Strub et al., 2006). The BRDF describes how the incident irradiance $F_{\mathrm{i}}$ from one direction $\left(\theta_{\mathrm{i}}, \varphi_{\mathrm{i}}\right)$ is reflected by a surface or a cloud into the direction $\left(\theta_{\mathrm{r}}, \varphi_{\mathrm{r}}\right)$. Here, $F_{\mathrm{i}}=\cos \theta_{\mathrm{i}} \cdot F_{0, \mathrm{i}}$ refers to a horizontal surface. With the reflected radiation being the radiance $I_{\mathrm{r}}\left(\theta_{\mathrm{r}}, \varphi_{\mathrm{r}}\right)$, the BRDF in units of $\mathrm{sr}^{-1}$ is defined by:

$\operatorname{BRDF}\left(\theta_{\mathrm{i}}, \varphi_{\mathrm{i}} ; \theta_{\mathrm{r}}, \varphi_{\mathrm{r}}\right)=\frac{\mathrm{d} I_{\mathrm{r}}\left(\theta_{\mathrm{i}}, \varphi_{\mathrm{i}} ; \theta_{\mathrm{r}}, \varphi_{\mathrm{r}}\right)}{\mathrm{d} F_{\mathrm{i}}\left(\theta_{\mathrm{i}}, \varphi_{\mathrm{i}}\right)}$.

In the literature, the dimensionless bidirectional reflectance factor BRF is often used instead of BRDF. It is defined as the ratio of the radiance $I_{\mathrm{r}}$ actually reflected by a surface to the radiance $I_{\mathrm{r}, \mathrm{L}}$ reflected by an ideal (non-absorbing) and diffuse (Lambertian) standard surface for identical irradiation and beam-geometry. An ideal Lambertian surface reflects the radiation isotropically, and it holds that $\mathrm{BRDF}_{\mathrm{L}}=$ $(\pi \mathrm{sr})^{-1}$. This results in the definition of the BRF:

$\operatorname{BRF}\left(\theta_{\mathrm{i}}, \varphi_{\mathrm{i}} ; \theta_{\mathrm{r}}, \varphi_{\mathrm{r}}\right)=\pi \mathrm{sr} \cdot \operatorname{BRDF}\left(\theta_{\mathrm{i}}, \varphi_{\mathrm{i}} ; \theta_{\mathrm{r}}, \varphi_{\mathrm{r}}\right)$.

However, both BRDF and BRF can be measured directly only when an artificial radiation source is applied. We present measurements in atmospheric conditions where the surface is illuminated by the Sun $\left(F_{\mathrm{i}}=F_{\mathrm{dir}}=\cos \theta_{0} \cdot F_{0}\right.$, $\left.\theta_{\mathrm{i}}=\theta_{0}, \varphi_{\mathrm{i}}=\varphi_{0}\right)$ and by diffuse radiation $\left(F_{\mathrm{diff}}\right)$. Both components give the global irradiance $F_{\text {glob }}=F_{\text {dir }}+F_{\text {diff. }}$. In this case, the hemispherical-directional reflectance factor HDRF is measured (Schaepman-Strub et al., 2006):

$\operatorname{HDRF}\left(\theta_{0}, \varphi_{0} ; 2 \pi ; \theta_{\mathrm{r}}, \varphi_{\mathrm{r}}\right)=\pi \mathrm{sr} \cdot \frac{\mathrm{d} I_{\mathrm{r}}\left(\theta_{0}, \varphi_{0} ; 2 \pi ; \theta_{\mathrm{r}}, \varphi_{\mathrm{r}}\right)}{\mathrm{d} F_{\text {glob }}\left(\theta_{0}, \varphi_{0}\right)}$.

Using the definition of $F_{\text {glob }}$ and introducing the fraction of direct incident radiation $f_{\text {dir }}=F_{\text {dir }} /\left(F_{\text {dir }}+F_{\text {diff }}\right)$, Eq. (8) can be transformed to:

$$
\begin{array}{r}
\operatorname{HDRF}\left(\theta_{0}, \varphi_{0} ; 2 \pi ; \theta_{\mathrm{r}}, \varphi_{\mathrm{r}}\right)=f_{\text {dir }} \cdot \operatorname{BRF}\left(\theta_{0}, \varphi_{0} ; \theta_{\mathrm{r}}, \varphi_{\mathrm{r}}\right) \\
+\left(1-f_{\text {dir }}\right) \cdot \operatorname{BRF}\left(2 \pi ; \theta_{\mathrm{r}}, \varphi_{\mathrm{r}}\right) .
\end{array}
$$



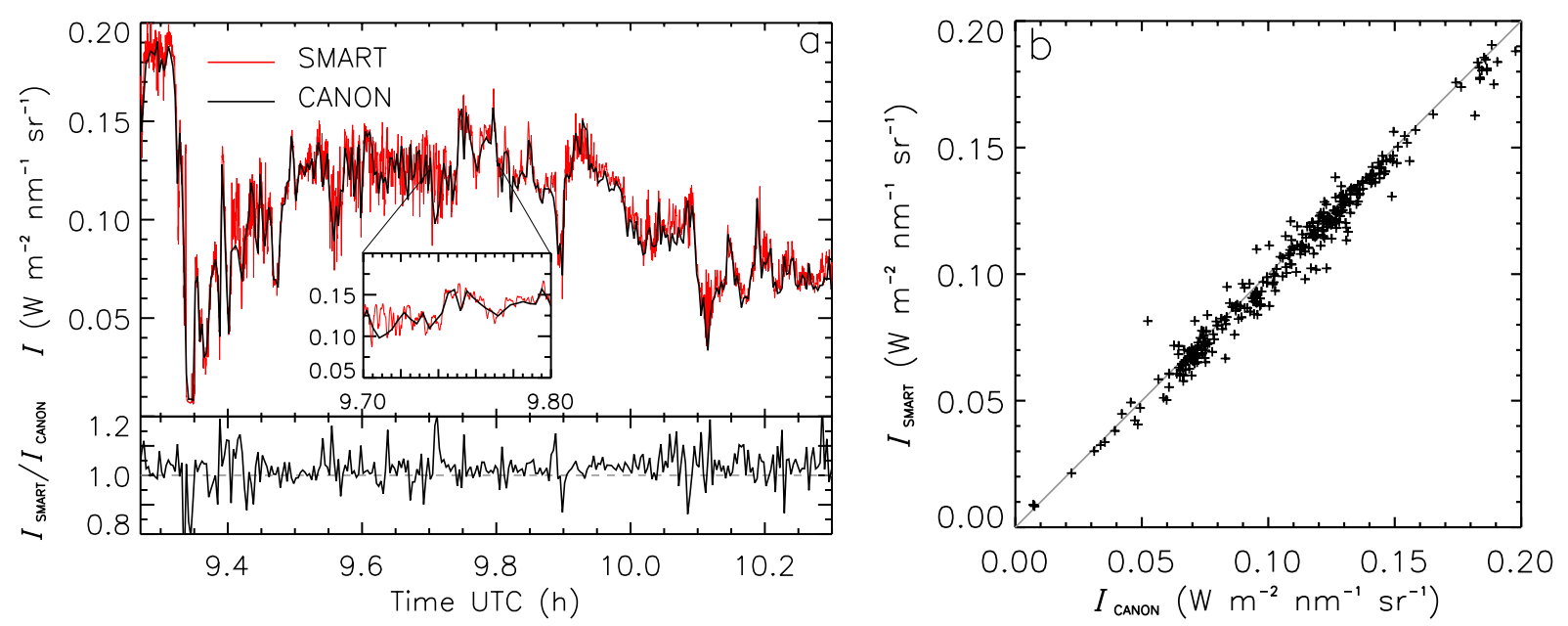

Fig. 5. Time series (17 May 2010) comparison (a) of a spectral radiance measured by the SMART-Albedometer (red) and by the CANON camera (black). Data are shown for the red camera channel $(\lambda=591 \mathrm{~nm})$. The correlation between both measurements is illustrated in second panel (b).

The measured HDRF can be split into the $\operatorname{BRF}\left(\theta_{0}, \varphi_{0} ; \theta_{\mathrm{r}}, \varphi_{\mathrm{r}}\right)$ for illumination of the surface by the Sun and the $\operatorname{BRF}\left(2 \pi ; \theta_{\mathrm{r}}, \varphi_{\mathrm{r}}\right)$ for pure diffuse illumination of the surface. Both components are weighted with $f_{\text {dir }}$, the fraction of direct incident radiation.

From HDRF measurements at a certain altitude, the BRDF, BRF, and the HDRF at surface level can be derived by applying an atmospheric correction using radiative transfer simulations, as shown by Gatebe et al. (2003) and Lyapustin et al. (2010). With the intention to validate the radiance and HDRF measurements of the camera as they are (at flight altitude), we do not apply an atmospheric correction for the comparison of measurements and simulations and present HDRF measurements instead of atmospherically corrected BRDF or BRF.

\section{Measurements}

\subsection{Spectral radiance}

By applying the radiometric calibration, the camera provides spectral radiances for each pixel and camera channel. The accuracy of the calibration was verified by comparing the nadir radiance of the camera to spectral measurements of the SMART-Albedometer, which has an uncertainty of $6 \%$ for radiance measurements (Ehrlich et al., 2008). The radiance optical inlet of the SMART-Albedometer is horizontally stabilized into nadir direction and has a field of view of $2.1^{\circ}$. This spot corresponds to about 16000 pixels of each camera image. These nadir pixels were averaged for each image. Furthermore, the spectral data of the SMART-Albedometer was adapted to the camera measurements by convolving the relative spectral response functions of the three camera channels (see Sect. 3.2).

In Fig. 5a, measurements of channel 1 are compared for an exemplary time interval on 17 May 2010, which were chosen to cover different surfaces such as sea ice, open ocean, and clouds. Despite the lower temporal resolution of the camera measurements (one image within $12 \mathrm{~s}$ ), the time series of radiances obtained from the camera images agree with the SMART-Albedometer measurements (temporal resolution of about $1 \mathrm{~s}$ ). The mean value of the SMARTAlbedometer measurements between 09:28 and 10:28 UTC is $\bar{I}=0.108 \mathrm{~W} \mathrm{~m}^{-2} \mathrm{~nm}^{-1} \mathrm{sr}^{-1}$, while the camera observed a mean nadir radiance of $\bar{I}=0.104 \mathrm{~W} \mathrm{~m}^{-2} \mathrm{~nm}^{-1} \mathrm{sr}^{-1}$. This difference of $4 \%$ ranges in the uncertainties range of the radiometric calibration of both instruments. As illustrated by the ratio of both measurements in the lower panel of Fig. 5a, the single data points differ more due to a non-perfect temporal allocation (integration times and sampling frequency) which makes averaging necessary. The standard deviation between both data sets is $0.006 \mathrm{~W} \mathrm{~m}^{-2} \mathrm{~nm}^{-1} \mathrm{sr}^{-1}$ with a correlation coefficient of 0.99 (see Fig. 5b). For the other spectral channels (not shown here), a similar behavior was observed, with differences in the mean values of $1 \%$ for channel 2 and $2 \%$ for channel 3. Standard deviation and correlation coefficient are almost identical for all channels. The agreement between both instruments shows that the CANON camera is capable of quantitatively measuring the distribution of reflected radiances, which can be used to derive the HDRF. In the following, results will be shown for channel 2 $(530 \mathrm{~nm})$ only. Channel 2 was chosen because it shows the smallest differences $(1 \%)$ to the SMART-Albedometer data and has the lowest electronic noise, as discussed in Sect. 3.3. 
Table 1. HDRF measurements above sea ice, open water, and clouds.

\begin{tabular}{lcccccccc}
\hline & Date & Time & Location & Altitude & \# of Images & $\theta_{0}$ & $\varphi_{0}$ & $\rho(530 \mathrm{~nm})$ \\
\hline Sea Ice & 14 May & $08: 21-08: 33$ & $82^{\circ} 00^{\prime} \mathrm{N}, 2^{\circ} 00^{\prime} \mathrm{W}$ & $100 \mathrm{~m}$ & 46 & $67.0^{\circ}$ & $123.0^{\circ}$ & 0.96 \\
Open Water & 14 May & $10: 21-10: 23$ & $79^{\circ} 20^{\prime} \mathrm{N}, 10^{\circ} 00^{\prime} \mathrm{E}$ & $3050 \mathrm{~m}$ & 11 & $61.1^{\circ}$ & $165.5^{\circ}$ & 0.12 \\
Clouds & 17 May & $09: 49-09: 59$ & $75^{\circ} 20^{\prime} \mathrm{N}, 18^{\circ} 30^{\prime} \mathrm{E}$ & $3100 \mathrm{~m}$ & 50 & $56.5^{\circ}$ & $166.5^{\circ}$ & 0.62 \\
\hline
\end{tabular}

\subsection{HDRF examples}

Images of the CANON camera were analyzed for three cases: sea ice, open water, and clouds. The HDRF was calculated using Eq. (8). The downward irradiance $F_{\text {glob }}\left(\theta_{0}, \varphi_{0}\right)$ was obtained from measurements of the SMART-Albedometer. The time and position of the observations, the corresponding position of the Sun, and the number of images used to build the averaged HDRFs are given in Table 1. The measurements above sea ice and open water were conducted on 14 May. The cloud scene was part of the measurements on 17 May, which were analyzed in Sect. 4.1. Using the irradiance measurements of the SMART-Albedometer, we additionally calculated the spectral albedo for each case. The measured albedo corresponding to the $530 \mathrm{~nm}$ channel of the camera is given in Table 1. For sea ice the albedo reaches a mean value of $\rho=0.96$. Above open water and clouds, $\rho=0.12$ and $\rho=0.62$, respectively, were observed.

The mean HDRF of each case is shown in Fig. 6 for camera channel 2. Additionally, a single characteristic image of the observed surface is shown.

\subsubsection{Sea ice}

Similarly to the albedo, the highest HDRF with values exceeding 1.0 was observed for sea ice, which was almost completely covered by snow. The measurements were conducted during the release of the towed EM-bird sonde, which observed a mean sea ice thickness of $2.5 \mathrm{~m}$. Therefore, the rope of the sonde was present in all images slightly affecting the HDRF measurements. Furthermore, ice ridges, as shown in Fig. 6a, have been frequently observed on the sea ice, showing a high contrast in the reflected radiance between shadow and illuminated areas. These horizontal inhomogeneities remain partly present in the mean HDRF calculated from 46 single images. However, the HDRF shows an almost Lambertian-like pattern, with only slight variability between 0.95 and 1.10 in the magnitude of HDRF. The lowest values are observed for nadir direction. The weak anisotropy with increasing HDRF along the principal plane is slightly stronger in the direction of the Sun (up to 1.1) than in the opposite direction (up to 1.0). For a similar solar zenith angle of $\theta_{0}=67^{\circ}$, Lyapustin et al. (2010) showed that the hot spot of the Sun influences the measured HDRF for zenith angles higher than $30^{\circ}$ along the principal plane. This is in agree- ment with the camera measurements covering zenith angles up to about $60^{\circ}$.

\subsubsection{Sea water}

Due to the high altitude at which the measurements above open water have been conducted, an atmospheric correction has been applied to extract the contribution of radiation reflected by the atmosphere below the aircraft. We adapted the iterative correction method by Wendisch et al. (2004) for the radiance measurements of the camera assuming a Lambertian-reflecting surface in the radiative transfer simulations.

Compared to the sea ice, the camera measurements above open water show a non-Lambertian pattern dominated by sun glint. In general, the HDRF of sea water (with minimum values of about 0.02 ) is significantly lower than for the sea ice and cloud case, which agrees with the low albedo. The sun glint area, which was only partly covered by the camera, shows values of up to 0.4 . The maximum of the sun glint (specular reflection for $61^{\circ}$ ) ranges outside the camera angle of view and might have even higher values. As discussed by Cox and Munk (1954), sun glint is caused by specular reflection at the surface waves, which is visible in the individual camera image of Fig. 6d. The surface wind measured by a drop sonde during the observations had a speed of about $9 \mathrm{~m} \mathrm{~s}^{-1}$ with a northerly direction $\left(360^{\circ}\right)$.

Compared to the sea ice and cloud measurements, the open water measurements required fewer images (11) for averaging because the sea surface is more homogeneous (as seen from about $3000 \mathrm{~m}$ altitude) than for the sea ice and cloud observations.

\subsubsection{Clouds}

The HDRF of a representative cloud was derived from measurements (50 individual images) above a low-level stratus cloud layer. For the area covered by the camera, the cloud HDRF ranges between 0.45 and 0.8 for the area covered by the camera. The anisotropy of the cloud HDRF mainly reflects the anisotropy of the scattering phase function of the cloud particles. For liquid water droplets, the scattering phase function has a maximum in forward scattering direction which explains the increasing HDRF in the direction of the Sun. The minimum values in both HDRF and scattering phase function are observed for the broad range 

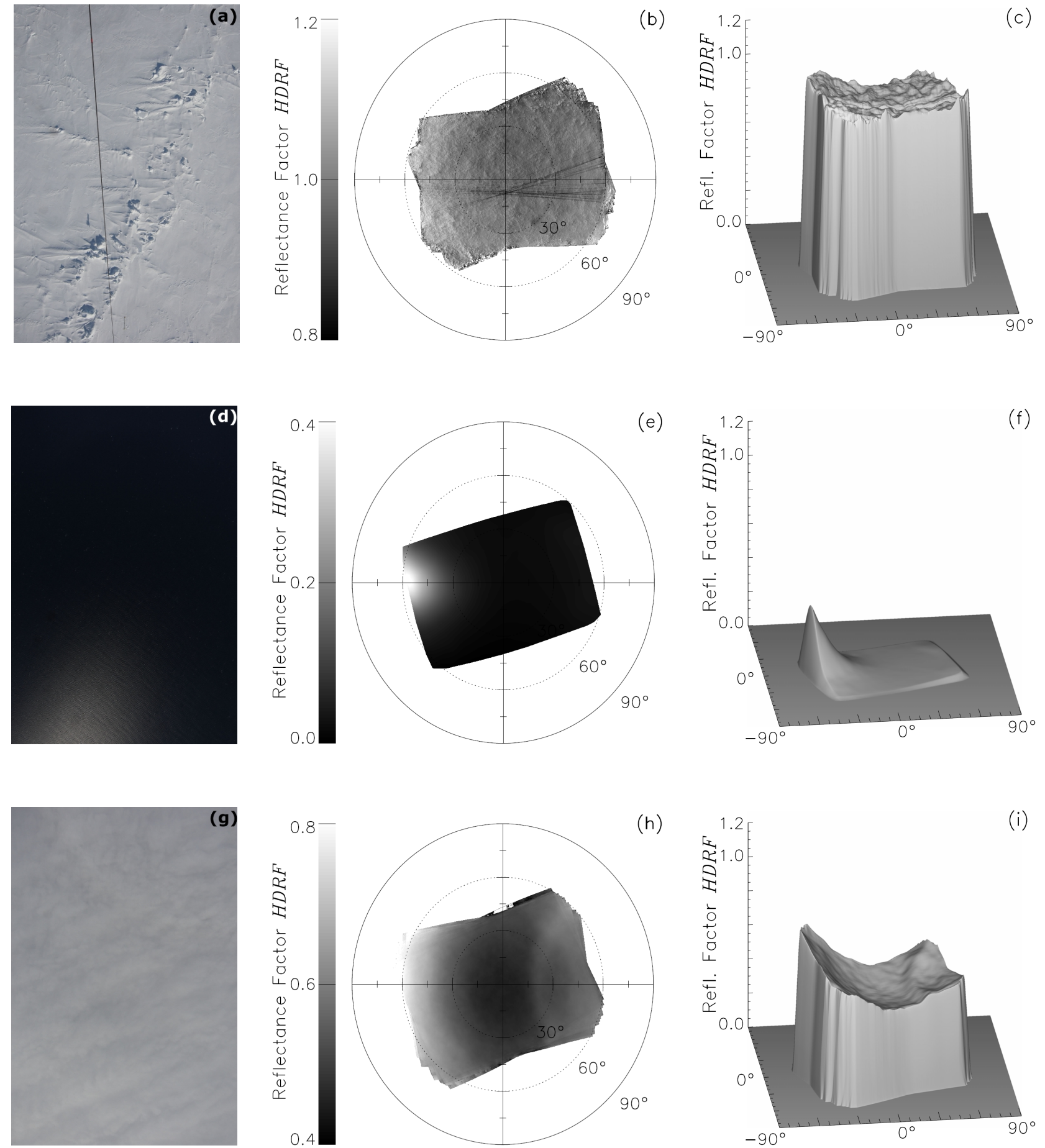

Fig. 6. HDRF measurements with the CANON camera (channel 2) above sea ice (top, $\theta_{0}=67.0^{\circ}$ ), open water $\left(\right.$ center, $\theta_{0}=61.1^{\circ}$ ), and clouds (bottom, $\theta_{0}=56.5^{\circ}$ ). The left column (a, d, g) shows exemplary individual camera images. The averaged HDRF is shown in the center column as polar plot (b, e, h) and as smoothed surface in the right column (c, e, i).

of sideways scattering $\left(\vartheta=80-120^{\circ}\right)$, which corresponds to the nadir direction of the camera measurements for solar zenith angle $\theta_{0}=56.5^{\circ}$. In the backscatter region, the scattering phase function of spherical particles shows local maxima (glory $\vartheta>175^{\circ}$ and cloudbow $\vartheta \approx 138^{\circ}$ ). While the glory was not covered by the camera, the cloudbow is visible in the measured HDRF as a ring around the backscatter point $\left(\theta_{\mathrm{r}}=\theta_{0}=56.5^{\circ}\right)$. 


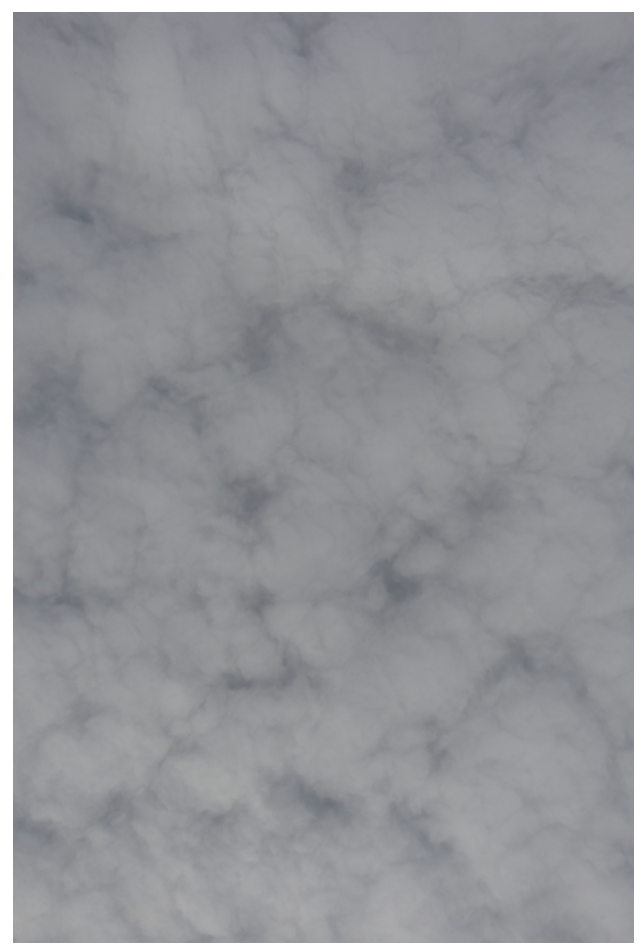

Fig. 7. Exemplary single camera image (09:41:23 UTC) of stratocumulus observations used to analyze averaging requirements.

\subsection{Averaging}

Due to inhomogeneities of the observed scene, the camera images had to be averaged to obtain a representative HDRF measurement. In the above examples, all available images (46, 11 and 50) were averaged for the sea ice, open water and cloud case, respectively. Especially for clouds, the narrow patterns of the glory and the cloudbow are visible in a single image only if the clouds are highly homogeneous. In most cases, even cloud layers such as stratocumulus have small-scale inhomogeneities which disturb the view of the glory and cloudbow. Therefore, averaging of several images was required to remove cloud inhomogeneities in the HDRF measurements.

For airborne measurement with POLDER, Descloitres et al. (1998) showed that after averaging a sequence of cloud observations, the scene acts like a plane-parallel cloud. The averaging approach assumes that the temporal cloud variability observed by each pixel in a sequence of images is similar to the spatial variability of one single image. Descloitres et al. (1998) found that about 10 images are required to sufficiently reduce the spatial variability for the observed cloud cases.

In a similar way, we investigated how many images are needed for sufficient averaging for the cloud observed during SORPIC. Data were analyzed for a typical stratocumulus observed on 17 May, 09:32 to 09:42 UTC. A single image
Table 2. Standard deviation $\sigma_{15^{\circ}}$ of the mean HDRF using 5, 10 , 20 or 50 images calculated for a circle of zenith angles lower than $15^{\circ}$. Additionally, $\sigma_{15^{\circ}}$ is given for a plane-parallel cloud of optical thickness $\tau=12$ and particle effective radius $R_{\text {eff }}=10 \mu \mathrm{m}$.

\begin{tabular}{cccccc}
\hline HDRF & 5 Images & 10 Images & 20 Images & 50 Images & Simulation \\
\hline$\sigma_{15^{\circ}}$ & 0.014 & 0.014 & 0.010 & 0.009 & 0.010 \\
\hline
\end{tabular}

illustrating the inhomogeneous horizontal cloud structure of the stratocumulus is shown in Fig. 7. The mean HDRFs for averaging 5, 10, 20 and 50 images are shown in Fig. 8 for channel 2. With a sampling frequency of one image for each $12 \mathrm{~s}$, this corresponds to flight times of $1 \mathrm{~min}, 2 \mathrm{~min}, 4 \mathrm{~min}$ and $10 \mathrm{~min}$. The plots show that for averaging 5 and $10 \mathrm{im}$ ages, the cloud structure is still visible in the mean HDRF. Using more images (20 and 50), the cloud structure begins to vanish but the cloudbow becomes more pronounced in the mean HDRF. This implies that for the stratocumulus case investigated here, averaging of about 50 images or more is necessary to obtain a HDRF in which the scattering phase function of the cloud droplets dominates the mean HDRF compared to cloud inhomogeneities. The number of 50 images is limited to this single case study only and may significantly differ for clouds with stronger inhomogeneity and observations at different altitudes. A stronger inhomogeneity would require more images to be averaged. On the other hand, images taken close to cloud top (not shown here) indicated the glory and cloudbow already in one single image.

To quantify the inhomogeneity of the HDRF, the standard deviation $\sigma_{15^{\circ}}$ of the HDRF was calculated for a circle of zenith angles lower than $15^{\circ}$ (about 70000 camera pixels). This narrow area was chosen to ensure that the standard deviation is not affected by the cloudbow at zenith angles larger than $15^{\circ}$. Before calculating $\sigma_{15^{\circ}}$, the HDRF was filtered by a 2-D low pass filter using an averaging window of $50 \times 50$ pixels. The filter removes the electronic noise in the images, which would also have been reduced by the averaging of images and thus biased $\sigma_{15^{\circ}}$. The filter window of $50 \times 50$ pixels is smaller than the natural cloud homogeneities and thus separates the effects of the electronic noise and natural cloud inhomogeneities. The values of $\sigma_{15^{\circ}}$ calculated for the mean HDRF of 5, 10, 20, and 50 images are given in Table 2. The values decrease with increasing number of images - from $\sigma_{15^{\circ}}=0.014$ for 5 images to $\sigma_{15^{\circ}}=0.009$ for averaging 50 images. In general, $\sigma_{15^{\circ}}$ does not converge to zero with increasing number of images, which is due to the anisotropy of the theoretical $\mathrm{HDRF}$ in the $15^{\circ}$ circle. To estimate the range of $\sigma_{15^{\circ}}$ for a perfectly homogeneous cloud, radiative transfer simulations were performed. For a cloud of optical thickness of $\tau=12$ and particle effective radius of $R_{\text {eff }}=10 \mu \mathrm{m}$, the simulations give a $\sigma_{15^{\circ}}$ of 0.01 . This ideal value ranges above $\sigma_{15^{\circ}}=0.009$ obtained for the mean HDRF using 50 images. This contradiction can only be explained by general 

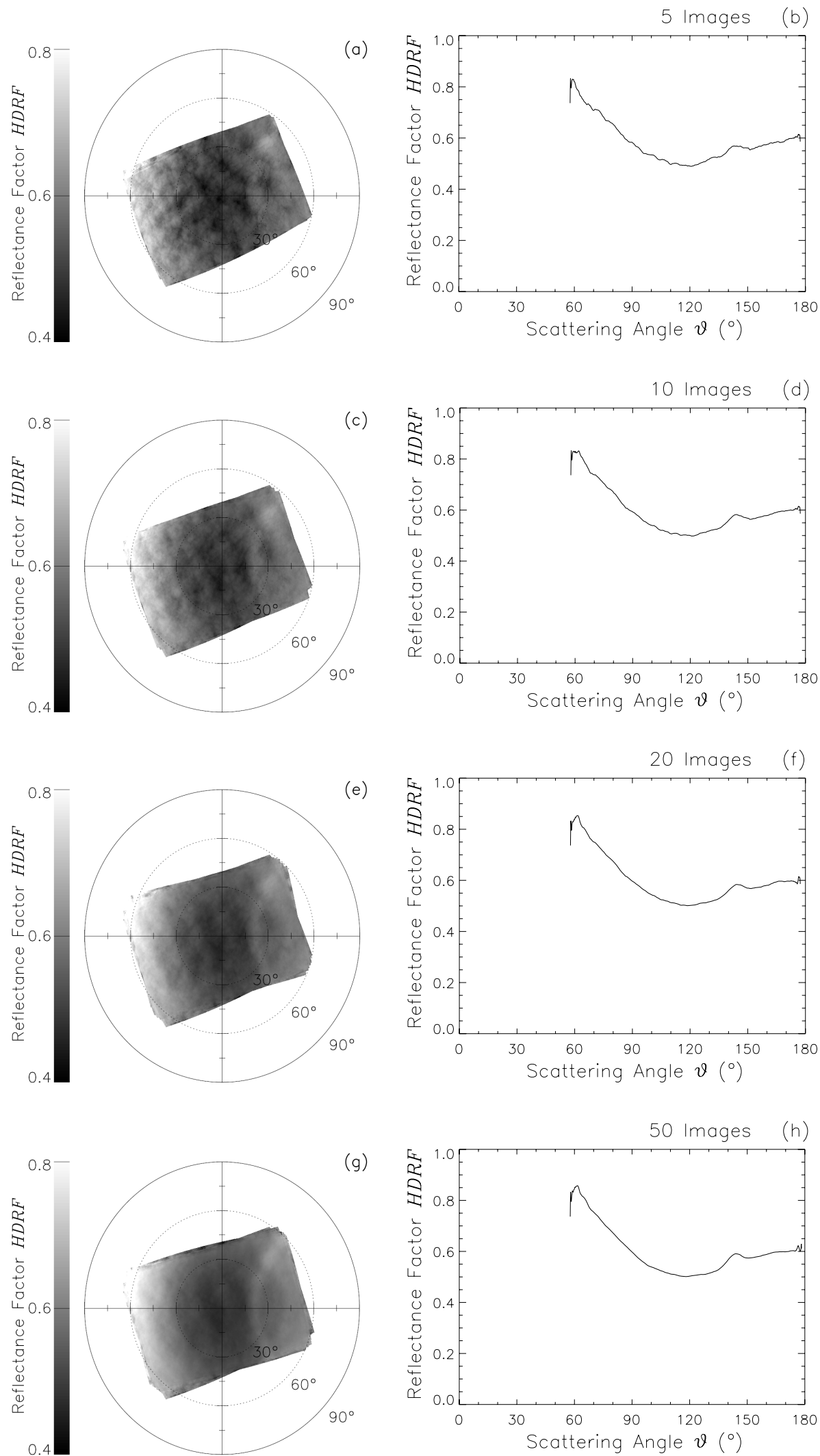

Fig. 8. Average HDRF above clouds (channel 2) calculated from 5, 10, 20, and 50 individual images (a, c, e, g). The right panels (b, d, f, h) show the corresponding $\operatorname{HDRF}(\vartheta)$ as function of the scattering angle. 
differences of the measured and simulated HDRF, but shows that an average of 50 images is sufficient to eliminate cloud inhomogeneities for the case presented here.

A way to reduce the required number of images is to present the HDRF as a function of the scattering angle $\vartheta$. Assuming that the scattering at homogeneous surfaces is rotationally symmetric with respect to the solar zenith angle, each image can be translated from the $\operatorname{HDRF}\left(\theta_{0}, \varphi_{0} ; \theta_{\mathrm{r}}, \varphi_{\mathrm{r}}\right)$ defined by the solar and viewing zenith and azimuth angles into a $\operatorname{HDRF}(\vartheta)$ defined by the scattering angle $\vartheta$. This transformation allows the averaging of several image pixels into one HDRF value for the corresponding $\vartheta$. We calculated $\operatorname{HDRF}(\vartheta)$ with a resolution of $0.1^{\circ}$. For each incremental scattering angle $\Delta \vartheta=0.1^{\circ}$, about 10000 pixels were averaged. In this way, the electronic noise of the camera sensor and the horizontal cloud inhomogeneities are smoothed more efficiently. The corresponding mean $\operatorname{HDRF}(\vartheta)$ for averaging 5, 10,20, and 50 images are shown in the right panels of Fig. 8 for channel 2. The $\operatorname{HDRF}(\vartheta)$ shows much less variability due to cloud inhomogeneities compared to the mean HDRF shown in the left panels of Fig. 8. The cloudbow can be already identified in the mean of 5 images. Averaging 10 images or more, the horizontal cloud inhomogeneities have been removed almost completely.

Although the average of 50 images indicates the backscatter glory for scattering angles larger than $176^{\circ}$, the glory was not perfectly covered on most images, being situated at the edge of the images. Therefore, the glory was not analyzed in the following.

\section{Simulated HDRF}

For the measurements above open water and above clouds, the HDRF was simulated by one-dimensional plane-parallel radiative transfer calculations. The simulations were run with the library for radiative transfer libRadtran by Mayer and Kylling (2005) using the discrete ordinate radiative transfer solver DISORT version 2.0 by Stamnes et al. (1988). The meteorological input (profiles of static air temperature, relative humidity, and static air pressure) was obtained from the drop sound released from Polar 5 at 10:25 UTC, 14 May for the open water case and 09:36 UTC, 17 May for the cloud case.

Radiances were calculated for the entire lower hemisphere. For one half of the cloud case with viewing direction into the Sun and for the entire open water case (where the HDRF is more homogeneous), the angular resolution was $5^{\circ}$ for the azimuth angle and $3^{\circ}$ for the zenith angle. The second half of the cloud case, including the glory and cloudbow in the backscattering region, was simulated with a higher angular resolution of $0.5^{\circ}$ for both angles. The results have been interpolated to the same grid as obtained by the camera measurements to allow a direct comparison.

\subsection{Open water}

The BRDF of sea water calculated by libRadtran is based on the parametrization of Cox and Munk (1954) and Nakajima and Tanaka (1983). The magnitude of the sun glint and the shape of the BRDF are mainly determined by the surface wind speed. Therefore, the parametrization was adjusted to the surface wind speed measured by the drop sonde. To analyze the sensitivity of the simulations with respect to the wind speed, three simulations with $5 \mathrm{~m} \mathrm{~s}^{-1}, 9 \mathrm{~m} \mathrm{~s}^{-1}$, and $15 \mathrm{~m} \mathrm{~s}^{-1}$ were performed, with $9 \mathrm{~m} \mathrm{~s}^{-1}$ being the value measured during the observations by a drop sonde. The wind direction was set to a northerly direction $\left(360^{\circ}\right.$,) corresponding to the observations. For the pigment concentration and the salinity, default values $\left(0.01 \mathrm{mg} \mathrm{m}^{-3}\right.$ for pigment concentration and $0.1 \mathrm{ppt}$ for salinity) were used. The simulations were performed for both surface and flight altitude to allow a direct comparison of the uncorrected measurement.

The result of the HDRF simulations with $9 \mathrm{~m} \mathrm{~s}^{-1}$ wind speed is shown in Fig. 9a for the flight altitude. The low values and the position of the sun glint agree with the measurements presented in Fig. 6e. In Fig. 9b, the absolute differences between measurements and simulations at flight altitude are given. For most of the areas covered by the camera measurements, the differences range below 0.01, indicated by the turquoise color. Only for the sun glint area at zenith angles larger than $45^{\circ}$ did the differences increase significantly and exceed values of -0.2 . The negative values show that in this area the simulations overestimate the HDRF compared to the measurements. Unfortunately, the sun glint is located at the outer edge of the image, where measurement uncertainties may increase due to a decreasing sensitivity of the camera sensor towards the sensor edges. However, an improper radiometric calibration of the camera can be excluded as reason for the deviations, as the differences occur only in the sun glint, while other boundary areas of the image agree well with the simulations.

Radiation reflected at angles similar to the sun glint is partially polarized, which may have affected the measurements for these scattering angles (Takashima, 1985). For the solar zenith angle $\left(61^{\circ}\right)$ and a scattering angle of about $60^{\circ}$, where maximum differences show up between simulated and measured $\operatorname{HDRF}(\vartheta)$, the degree of polarization may reach maximum values up to 0.9 (A. Hollstein, personal communication, 2011). However, the uncertainty of the camera due to polarization was estimated to be $3 \%$ at maximum and cannot completely explain the differences between measurements and simulations. The images were also checked for saturation. With a low exposure time (1/2656 s) adjusted to the bright scenes of clouds and sea ice, no saturation was evident in the data. The raw data of the images showed maximum digital counts of about 12000 in the sun glint and about 25000 counts for sea ice, with a saturation value of 65536 (16 bit). 

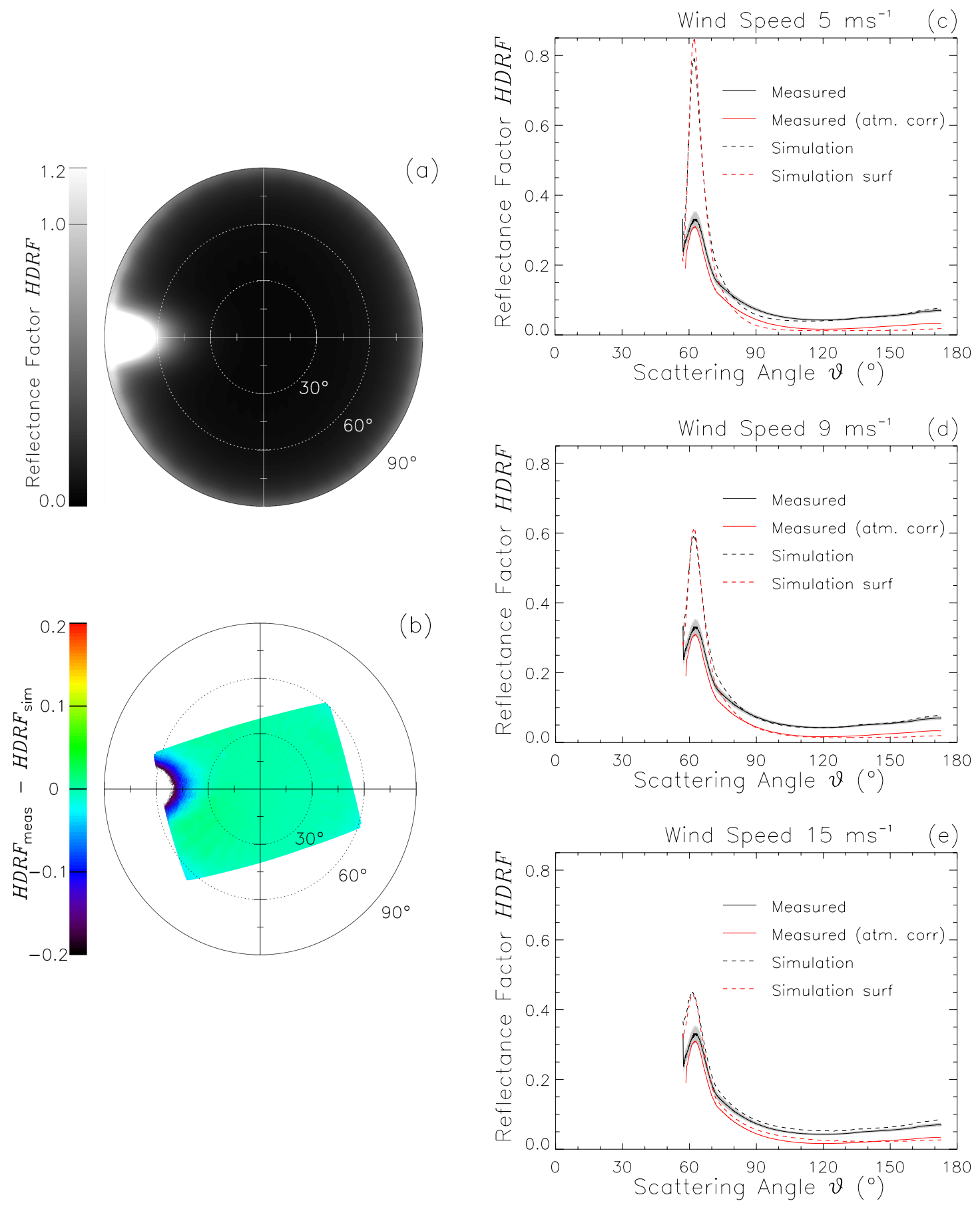

Fig. 9. Simulated HDRF of open water. The left panels show (a) the HDRF simulated with $9 \mathrm{~m} \mathrm{~s}^{-1}$ wind speed and (b) the difference to the measurements. The angular distribution of the HDRF is compared between measurements (solid line) and simulations (dashed line) with different wind speed in panels (c) $5 \mathrm{~m} \mathrm{~s}^{-1}$, (d) $9 \mathrm{~m} \mathrm{~s}^{-1}$ and (e) $15 \mathrm{~m} \mathrm{~s}^{-1}$. The uncertainty of the measurements is indicated by the gray area. The red solid and dashed lines represent the measured $\operatorname{HDRF}(\vartheta)$ after atmospheric correction and the $\operatorname{HDRF}(\vartheta) \operatorname{simulated}$ at the $\operatorname{surface}$, respectively.

The angular distribution $\operatorname{HDRF}(\vartheta)$ can be used to analyze the differences between simulations and measurements, as shown in Fig. $9 c-d$. From the simulations, results for the en- tire hemisphere are obtained. However, we only calculated $\operatorname{HDRF}(\vartheta)$ from the area which was covered by the camera images. The $\operatorname{HDRF}(\vartheta)$ of the entire hemisphere (not shown 

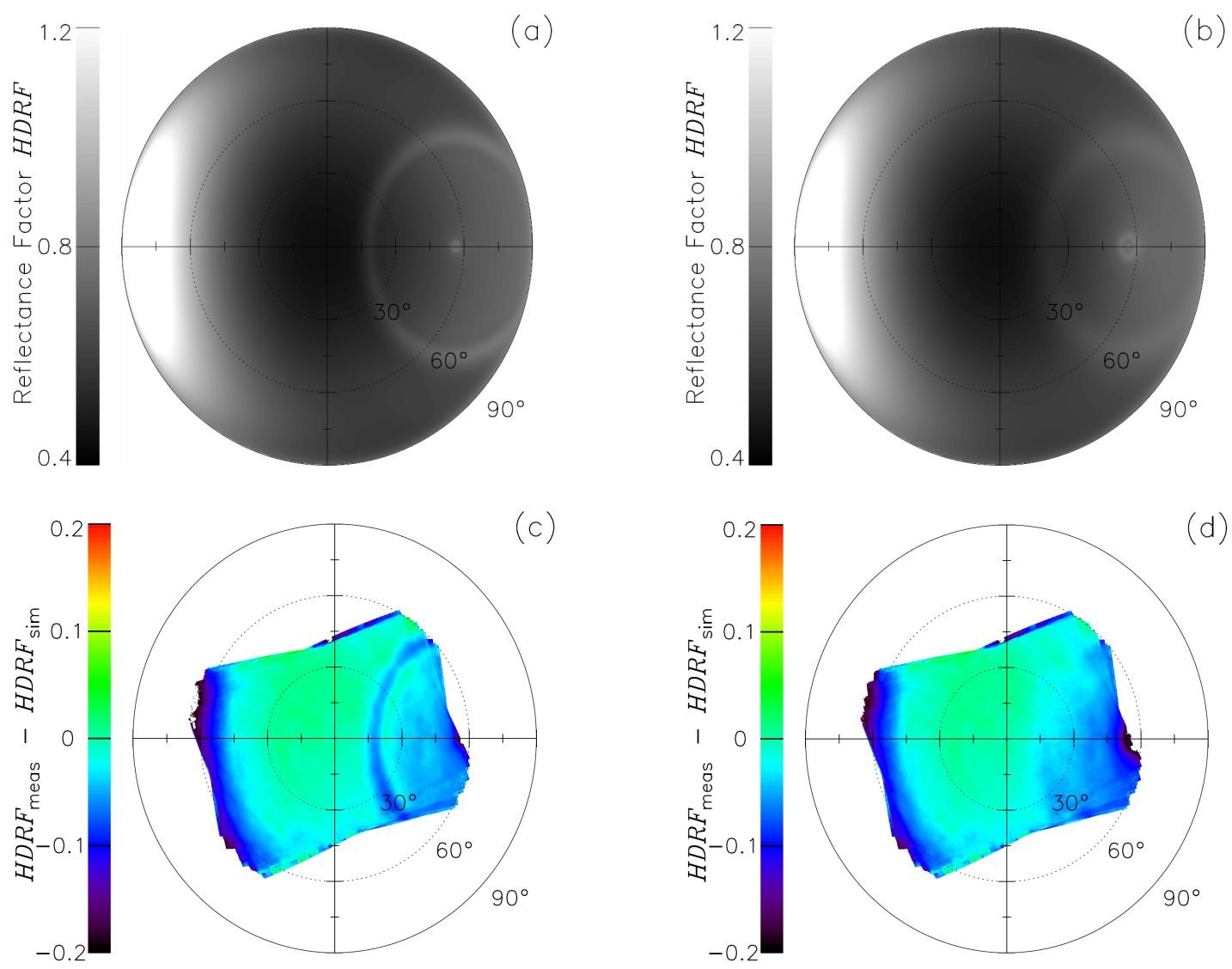

(e)
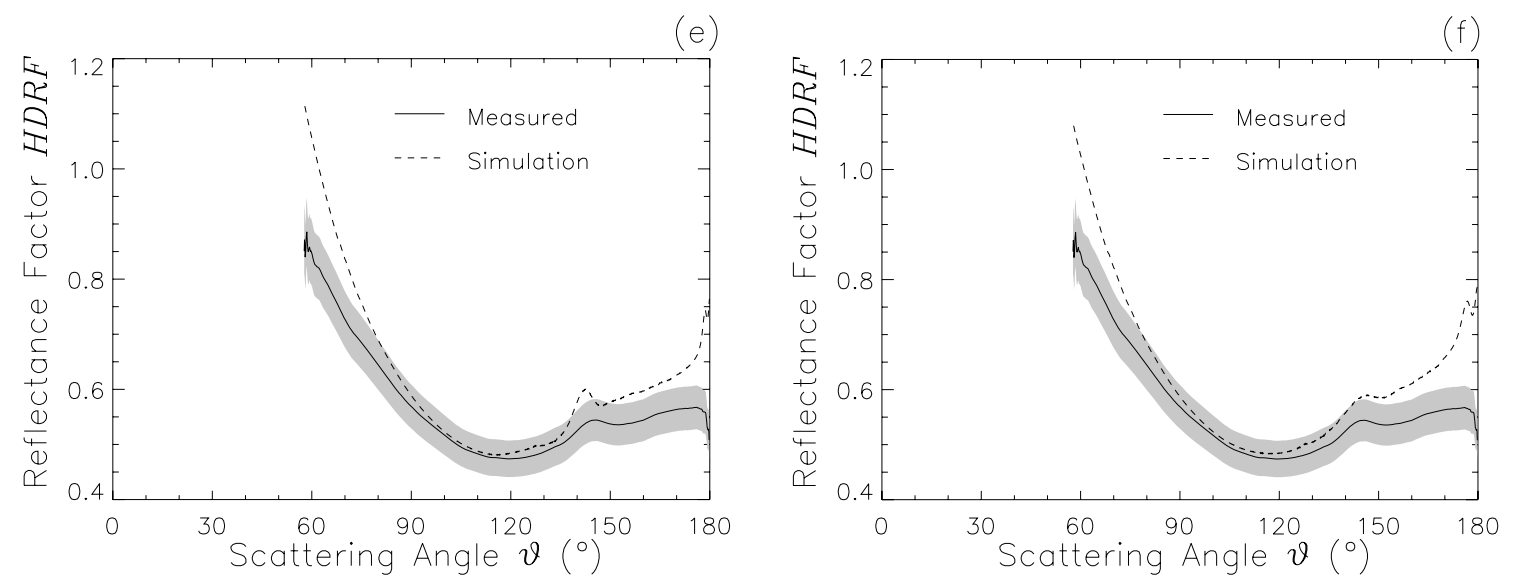

Fig. 10. Simulated HDRF for clouds with optical thickness and effective diameter of $\tau=12$ and $R_{\text {eff }}=10 \mu \mathrm{m}($ left $)$ and of $\tau=10, R_{\text {eff }}=$ $4 \mu \mathrm{m}$ (right). The upper panels show the HDRF of the entire lower hemisphere. Differences between measured and simulated HDRF are given in the middle panels $(\mathbf{c}, \mathbf{d})$. The lower panels $(\mathbf{e}, \mathbf{f})$ give the $\operatorname{HDRF}(\vartheta)$ as function of the scattering angle for measurements and simulations. The uncertainty of the measurements is indicated by the gray area.

here) differs significantly for most of the scattering angles because they include the viewing directions close to the horizon, where multiple scattering leads to enhanced reflection. Figure $9 \mathrm{c}-\mathrm{d}$ additionally shows the measurements corrected for the atmosphere and the simulations at surface altitude (red lines).
Comparing simulations and measurements of the selected area (Fig. 9d), the simulated $\operatorname{HDRF}(\vartheta)$ differs from the measurement for scattering angles lower than $80^{\circ}$, while for larger scattering angles they fit into the uncertainty range of the measurements. The position of the local sun glint maximum within the image is well covered by the measurements, 
but the magnitude differs by up to 0.25 . Similar differences are observed for the HDRF at surface altitude.

The HDRF of simulations carried out with surface wind speeds of $5 \mathrm{~m} \mathrm{~s}^{-1}$ and $15 \mathrm{~m} \mathrm{~s}^{-1}$ are shown in Fig. 9c and e. Compared to the HDRF using $9 \mathrm{~m} \mathrm{~s}^{-1}$ wind speed, it stands out that the maximum HDRF values, which are located in the sun glint area, decrease with increasing wind speed. The closest agreement with the measurements is obtained from the simulations with $15 \mathrm{~m} \mathrm{~s}^{-1}$ wind speed. However, while the simulations with $9 \mathrm{~m} \mathrm{~s}^{-1}$ wind speed fit to the measurements at flight altitude for all scattering angles larger than $80^{\circ}$, the simulated HDRF at $5 \mathrm{~m} \mathrm{~s}^{-1}$ and $15 \mathrm{~m} \mathrm{~s}^{-1}$ differ for these scattering angles. The HDRF simulated using a wind speed of $5 \mathrm{~m} \mathrm{~s}^{-1}$ ranges significantly below the measurements for scattering angles between $80^{\circ}$ and $120^{\circ}$, while the $15 \mathrm{~m} \mathrm{~s}^{-1}$ HDRF ranges above the measurements for all scattering angles. The higher HDRF values simulated for $15 \mathrm{~m} \mathrm{~s}^{-1}$ wind speed may result from an increase of white caps. The amount of white caps, which is correlated to the albedo of open water (Gordon and Jacobs, 1977), increases nearly linearly between $5 \mathrm{~m} \mathrm{~s}^{-1}$ and $15 \mathrm{~m} \mathrm{~s}^{-1}$, as shown by Stramska and Petelski (2003). A higher albedo is directly linked to a higher HDRF. Therefore, we argue that the measured HDRF correspond best to the observed wind speed of $9 \mathrm{~m} \mathrm{~s}^{-1}$, despite the differences in the sun glint area.

\subsection{Clouds}

To analyze the HDRF measured above clouds, radiative transfer calculations were used to simulate the cloud case observed on 17 May. The cloud optical properties required for the model input have been retrieved from SMARTAlbedometer measurements using the method introduced by Nakajima and King (1990). For the flight leg between 09:49 UTC and 09:59 UTC (see Table 1), the mean optical thickness was about $\tau=11.5$, with the cloud droplet effective radius $R_{\text {eff }}$ ranging between $4 \mu \mathrm{m}$ and $10 \mu \mathrm{m}$. The effective radius obtained from the in situ instrumentation about one hour after the remote sensing measurements was about $R_{\text {eff }}=9 \mu \mathrm{m}$.

Considering the variation of $R_{\mathrm{eff}}$, simulations for two clouds with $R_{\mathrm{eff}}=4 \mu \mathrm{m}$ and $R_{\mathrm{eff}}=10 \mu \mathrm{m}$ were performed. As $\tau$ and $R_{\text {eff }}$ are linked with each other, we adjusted $\tau$ to fit the simulated HDRF in nadir direction to the measurements of the SMART-Albedometer. For the cases of $R_{\text {eff }}=4 \mu \mathrm{m}$ and $R_{\text {eff }}=10 \mu \mathrm{m}$, the cloud optical thickness was scaled to $\tau=10.5$ and $\tau=12.0$, respectively. The spectral surface albedo is represented by SMART-Albedometer measurements above sea water obtained for similar conditions during the ASTAR 2007 campaign (Ehrlich et al., 2008). For this cloud case with a moderate cloud optical thickness, the albedo is sufficient to describe the surface reflectivity. The BRDF derived using the parametrization of Cox and Munk (1954) has not been applied, because additional simulations have shown no differences between simulations using the albedo or the BRDF.

For both simulations with $R_{\text {eff }}=4 \mu \mathrm{m}$ and $R_{\text {eff }}=10 \mu \mathrm{m}$, the HDRF is shown in Fig. 10 (upper panels). In both cases, the HDRF is characterized by the sun glint for zenith angles larger than $75^{\circ}$ in the direction of the Sun and the glory and cloudbow in the backscattering region. The lowest HDRF is simulated in the nadir direction. The comparison of both simulations indicates that the size of the glory decreases with increasing cloud droplet effective radius. The first order maximum is at $176.3^{\circ}$ scattering angle for $R_{\mathrm{eff}}=4 \mu \mathrm{m}$ and $\vartheta=178.4^{\circ}$ scattering angle for $R_{\text {eff }}=10 \mu \mathrm{m}$, respectively. Similarly, the characteristics of the cloudbow change with cloud droplet size. For simulations with larger droplet size, the cloudbow is more pronounced and the maximum is at a smaller scattering angle compared to a cloud with small droplets.

The simulations are compared to the HDRF derived from the camera measurements in the middle panels of Fig. 10, where the differences between measurements and simulations are shown. Positive differences (green and orange color) correspond to cases where the measurements showed higher values than calculated by the simulations. The blue color indicates negative differences where the measured values range below the simulations.

For both simulations the lowest differences are observed in nadir direction, which results from scaling the optical thickness with regard to the measured nadir radiance. Higher differences up to values of -0.2 are obtained for larger zenith angles. In the direction of the Sun, these differences are related to the sun glint where the HDRF is enhanced. Here, the simulation did calculate significantly higher HDRF values than observed by the camera. In the backscatter region, the differences are related to the glory and cloudbow, with maximum differences close to the $180^{\circ}$ point at $56^{\circ}$ zenith angle. Most striking are the differences corresponding to the cloudbow in the simulations for $R_{\mathrm{eff}}=10 \mu \mathrm{m}$. While the cloudbow pattern is visible for large droplets, the simulations for the smaller cloud droplets $\left(R_{\text {eff }}=4 \mu \mathrm{m}\right)$ do not significantly differ from the measurement. This indicates that the HDRF measurements can be used to characterize the cloud particle size.

A similar picture is obtained by comparing the angular distribution $\operatorname{HDRF}(\vartheta)$ of the limited area to the camera measurements, as illustrated in the lower panels of Fig. 10. Again, the simulations for $R_{\mathrm{eff}}=4 \mu \mathrm{m}$ fit better to the observations than the simulations using $R_{\text {eff }}=10 \mu \mathrm{m}$. The signature of the cloudbow with a broader and lower maximum is reproduced best if smaller cloud droplets are assumed in the simulations. The narrow and intense cloudbow simulated for the larger cloud droplets significantly exceeds the measured $\operatorname{HDRF}(\vartheta)$ at scattering angles around $142^{\circ}$.

The differences at small and large scattering angles below $80^{\circ}$ and above $150^{\circ}$ correspond to the margins of the camera images where the statistics are bad compared to the center of 
the images. Furthermore, 3-D effects may reduce the measured $\operatorname{HDRF}(\vartheta)$ compared to the simulations. As Loeb and Coakley Jr. (1998) and Loeb et al. (1998) have shown, the 3-D structure of clouds leads to a decreasing cloud reflectivity towards the horizon compared to one-dimensional planeparallel simulations which we applied here.

\section{Conclusions}

Images measured with a commercial digital single-lens reflex camera have been analyzed to produce the HDRF of different surfaces and clouds. For this purpose, the camera was calibrated spectrally and radiometrically. The central wavelengths of the three spectral channels are $591 \mathrm{~nm}$ (red), $530 \mathrm{~nm}$ (green), and $446 \mathrm{~nm}$ (blue) with a FWHM of about $80 \mathrm{~nm}$. The radiometric calibration showed a decreasing sensitivity towards the boundaries of the camera sensor, which is a typical vignetting effect of digital photo cameras (Lebourgeois et al., 2008). Dark current, polarization effects, and sensor saturation were found to be negligible for the measurement uncertainty. A comparison with spectral radiance measurements provided by the SMART-Albedometer shows differences below the uncertainty range of both instruments $(6 \%)$. This agreement shows that the CANON camera is capable of measuring calibrated radiances.

HDRF measurements were obtained for sea ice, open water, and clouds. In general, the results agree with known literature. Compared to traditional measurements, the high spatial resolution of the camera provides a detailed view on the angular pattern of the HDRF, including the sun glint of open water and the cloudbow for the cloud HDRF. However, to obtain a representative HDRF, averaging was necessary. Due to the high spatial resolution of the camera, small-scale inhomogeneities of the surface (sea ice or open water) or of the cloud were resolved by the observations and then averaged. For the inhomogeneous stratocumulus clouds analyzed here, the required number of images was estimated to be 50 . When the HDRF was translated into an angular distribution $\operatorname{HDRF}(\vartheta)$, the required number of images was reduced to 10 . With a sampling frequency of one image per $12 \mathrm{~s}$, these required numbers of 50 and 10 images correspond to sampling times of $10 \mathrm{~min}$ and $2 \mathrm{~min}$, respectively. These could be reduced if the maximum sampling frequency provided by the camera (one image in $6 \mathrm{~s}$ ) was applied. These numbers hold only for the clouds investigated here and may change for clouds with different inhomogeneity. Also, the flight altitude will alter the details resolved by the camera and thus the sampling time for one HDRF measurement.

For the measurements above open water and clouds, radiative transfer simulations providing HDRF were applied and compared to the measurements. Except for the sun glint region, the open-water case agreed well with the HDRF based on the BRDF parametrization of Cox and Munk (1954). The magnitude of the sun glint was simulated with higher values compared to the measurements. Simulations with a higher surface wind speed reduced the sun glint but also increased the HDRF outside the sun glint. Known measurement uncertainties like polarization effects, lens distortion and sensor saturation were estimated and ruled out as reasons for the differences. Further measurements with different surface wind conditions and solar zenith angles have to be analyzed to determine whether these differences are model or measurement based.

The measurements above clouds showed that the cloudbow can be extracted from the images. The position, magnitude, and width of the cloudbow agreed with simulations, assuming cloud droplets with an effective diameter $R_{\mathrm{eff}}=$ $4 \mu \mathrm{m}$. Simulations assuming $R_{\mathrm{eff}}=10 \mu \mathrm{m}$ failed to reproduce the observed cloudbow. This indicates that the analysis of the cloudbow could possibly be used to retrieve the cloud effective diameter. A similar approach was successfully applied by Mayer et al. (2004) who derived the particle size from analyzing the width of the backscatter glory. For ice clouds, multi-angle satellite measurements have been utilized by Chepfer et al. (2002) to retrieve the ice crystal shape. This method, based on differences in scattering phase functions of ice crystals, might be applied to our camera measurements in future. However, detailed analysis of the images and further observation of different clouds are necessary to obtain a reliable retrieval. Uncertainties in the aircraft attitude may broaden the cloudbow when the images are not perfectly corrected. Additionally, stereo effects for inhomogeneous clouds with varying cloud top height may broaden the cloudbow. This would lead to an underestimation of the cloud droplet size. However, HDRF above different clouds (not shown here) did show a narrower cloudbow, indicating larger cloud droplets. As it was not our intention to provide a retrieval method for the cloud effective radius, no detailed studies on radii are shown here.

The HDRF measurements presented here are limited to the field of view of the camera lens with a maximum of $100^{\circ}$ in the image diagonal. A circular flight pattern might be helpful to increase the angular coverage of the images. However, considering the required averaging, several circles would have to be flown, which would increase the sampling time of one HDRF measurement significantly. An alternative to improve the camera measurements would be the application of a $180^{\circ}$ field of view lens, which would enable us to cover the entire lower hemisphere within one single image. In this way, the full sun glint pattern and the entire backscatter glory would be covered and provide more detailed information on the surface and cloud microphysical properties.

Acknowledgements. This research was funded by the German Research Foundation (DFG, WE 1900/17-1) and AWI which generously provided flight hours, logistic support, and general access to POLAR 5 for the SORPIC campaign. For the technical support during SORPIC, we want to acknowledge the company Fielax and personally thank Martin Gehrmann of the AWI flight operations department. Further, we want to thank both reviewers 
for their excellent job. The huge number of comments did help a lot to improve the paper.

Edited by: T. Garrett

\section{References}

Buriez, J.-C., Parol, F., Cornet, C., and Doutriaux-Boucher, M.: An improved derivation of the top-of-atmosphere albedo from POLDER/ADEOS-2: Narrowband albedos, J. Geophys. Res., 110, D05202, doi:10.1029/2004JD005243, 2005.

Chepfer, H., Minnis, P., Young, D., Nguyen, L., and Arduini, R. F.: Estimation of cirrus cloud effective ice crystal shapes using visible reflectances from dual-satellite measurements, J. Geophys. Res., 107, 4730, doi:10.1029/2000JD000240, 2002.

Cox, C. and Munk, W.: Measurement of the roughness of the sea surface from photographs of the sun's glitter, J. Opt. Soc. Am. A., 44, 838-850, 1954.

Descloitres, J., Buriez, J. C., Parol, F., and Fouquart, Y.: POLDER observations of cloud bidirectional reflectances compared to a plane-parallel model using the International Satellite Cloud Climatology Project cloud phase functions, J. Geophys. Res.Atmos., 103, 11411-11418, doi:10.1029/98JD00592, 1998.

Dumont, M., Brissaud, O., Picard, G., Schmitt, B., Gallet, J.-C., and Arnaud, Y.: High-accuracy measurements of snow Bidirectional Reflectance Distribution Function at visible and NIR wavelengths - comparison with modelling results, Atmos. Chem. Phys., 10, 2507-2520, doi:10.5194/acp-10-2507-2010, 2010.

Ehrlich, A., Bierwirth, E., Wendisch, M., Gayet, J.-F., Mioche, G., Lampert, A., and Heintzenberg, J.: Cloud phase identification of Arctic boundary-layer clouds from airborne spectral reflection measurements: test of three approaches, Atmos. Chem. Phys., 8, 7493-7505, doi:10.5194/acp-8-7493-2008, 2008.

Gatebe, C., King, M., PLatnick, S., Arnold, G., Vermote, E., and Schmid, B.: Airborne spectral measurements of surface-atmosphere anisotropy for several surfaces and ecosystems over southern Africa, J. Geophys. Res., 108, 8489, doi:10.1029/2002JD002397, 2003.

Gatebe, C. K., King, M. D., Lyapustin, A. I., Arnold, G. T., and Redemann, J.: Airborne spectral measurements of ocean directional reflectance, J. Atmos. Sci., 62, 1072-1092, 2005.

Gayet, J.-F., Mioche, G., Dörnbrack, A., Ehrlich, A., Lampert, A., and Wendisch, M.: Microphysical and optical properties of Arctic mixed-phase clouds. The 9 April 2007 case study., Atmos. Chem. Phys., 9, 6581-6595, doi:10.5194/acp-9-6581-2009, 2009.

Gordon, H. R. and Jacobs, M. M.: Albedo of Ocean-atmosphere System - Influence of Sea Foam, Appl. Optics, 16, 2257-2260, 1977.

Haas, C., Lobach, J., Hendricks, S., Rabenstein, L., and Pfaffling, A.: Helicopter-borne measurements of sea ice thickness, using a small and lightweight, digital EM system, J. Appl. Geophys., 67, 234-241, doi:10.1016/j.jappgeo.2008.05.005, 2009.

Hyer, E. J., Reid, J. S., and Zhang, J.: An over-land aerosol optical depth data set for data assimilation by filtering, correction, and aggregation of MODIS Collection 5 optical depth retrievals, Atmos. Meas. Tech., 4, 379-408, doi:10.5194/amt-4-379-2011, 2011.
Kaufmann, K.: CMOS Technology for Scientific Imaging, Spectroscopy, 25, 20-25, 2010.

Lampert, A., Ehrlich, A., Dörnbrack, A., Jourdan, O., Gayet, J.F., Mioche, G., Shcherbakov, V., Ritter, C., and Wendisch, M.: Microphysical and radiative characterization of a subvisible midlevel Arctic ice cloud by airborne observations - a case study, Atmos. Chem. Phys., 9, 2647-2661, doi:10.5194/acp-9-26472009, 2009.

Lebourgeois, V., Bégué, A., Labbé, S., Mallavan, B., Prévot, L., and Roux, B.: Can commercial digital cameras be used as multispectral sensors? A crop monitoring test, Sensors, 8, 7300-7322, doi:10.3390/s8117300, 2008.

Litvinov, P., Hasekamp, O., and Cairns, B.: Models for surface reflection of radiance and polarized radiance: Comparison with airborne multi-angle photopolarimetric measurements and implications for modeling top-of-atmosphere measurements, Remote Sens. Environ., 115, 781-792, doi:10.1016/j.rse.2010.11.005, 2011.

Loeb, N. and Coakley Jr., J.: Inference of marine stratus cloud optical depths from satellite measurements: Does 1D theory apply?, J. Climate, 11, 215-233, 1998.

Loeb, N. and Davies, R.: Angular dependence of observed reflectances: A comparison with plane parallel theory, J. Geophys. Res., 102, 6865-6881, 1997.

Loeb, N., Varnai, T., and Winker, D.: Influence of subpixel-scale cloud-top structure of reflectances from overcast stratiform cloud layers, J. Atmos. Sci., 55, 2960-2973, 1998.

Loeb, N., Parol, F., Buriez, J.-C., and Vanbauce, C.: Top-ofatmosphere albedo estimation from angular distribution models using scene identification from satellite cloud property retrievals, J. Climate, 13, 1269-1285, 2000.

Loeb, N., Kato, S., Loukachine, K., and Manalo-Smith, N.: Angular Distribution Models for Top-of-Atmosphere Radiative Flux Estimation from the Clouds and the Earth's Radiant Energy System Instrument on the Terra Satellite. Part I: Methodology, J. Atmos. Ocean. Tech., 22, 338-351, 2005.

Long, C. N., Sabburg, J. M., Calbo, J., and Pages, D.: Retrieving cloud characteristics from ground-based daytime color allsky images, J. Atmos. Ocean. Tech., 23, 633-652, 2006.

Lyapustin, A., Gatebe, C. K., Kahn, R., Brandt, R., Redemann, J., Russell, P., King, M. D., Pedersen, C. A., Gerland, S., Poudyal, R., Marshak, A., Wang, Y., Schaaf, C., Hall, D., and Kokhanovsky, A.: Analysis of snow bidirectional reflectance from ARCTAS Spring-2008 Campaign, Atmos. Chem. Phys., 10, 4359-4375, doi:10.5194/acp-10-4359-2010, 2010.

Mayer, B. and Kylling, A.: Technical note: The libRadtran software package for radiative transfer calculations - description and examples of use, Atmos. Chem. Phys., 5, 1855-1877, doi:10.5194/acp-5-1855-2005, 2005.

Mayer, B., Schröder, M., Preusker, R., and Schüller, L.: Remote sensing of water cloud droplet size distributions using the backscatter glory: a case study, Atmos. Chem. Phys., 4, 12551263, doi:10.5194/acp-4-1255-2004, 2004.

Nakajima, T. and King, M.: Determination of the optical thickness and effective particle radius of clouds from reflected solar radiation measurements. Part I: Theory, J. Atmos. Sci., 47, 18781893, 1990.

Nakajima, T. and Tanaka, M.: Effect of wind-generated waves on the transfer of solar radiation in the atmosphere-ocean system, J. 
Quant. Spectrosc. Ra., 29, 521-537, 1983.

Nicodemus, F., Richmond, J., Hsia, J., Ginsber, I. W., and Limperis, T.: Geometrical Considerations and Nomenclature for Reflectance, vol. 160 of NBS Monograph, US Department of Commerce, Washington, D.C., National Bureau of Standards, 1977.

Olsen, D., Dou, C., Zhang, X., Hu, L., Kim, H., and Hildum, E.: Radiometric Calibration for AgCam, Remote Sensing, 2, 467477, doi:10.3390/rs2020464, 2010.

Ovtchinnikov, M. and Marchand, R. T.: Cloud model evaluation using radiometric measurements from the airborne multiangle imaging spectroradiometer (AirMISR), Remote Sens. Environ., 107, 185-193, doi:10.1016/j.rse.2006.05.024, 2007.

Schade, N. H., Macke, A., Sandmann, H., and Stick, C.: Total and partial cloud amount detection during summer 2005 at Westerland (Sylt, Germany), Atmos. Chem. Phys., 9, 1143-1150, doi:10.5194/acp-9-1143-2009, 2009.

Schaepman-Strub, G., Schaepman, M. E., Painter, T. H., Dangel, S., and Martonchik, J. V.: Reflectance quantities in optical remote sensing-definitions and case studies, Remote Sens. Environ., 103, 27-42, 2006.

Stamnes, K., Tsay, S., Wiscombe, W., and Jayaweera, K.: A numerically stable algorithm for discrete-ordinate-method radiative transfer in multiple scattering and emitting layered media, Appl. Optics, 27, 2502-2509, 1988.
Stramska, M. and Petelski, T.: Observations of oceanic whitecaps in the north polar waters of the Atlantic, J. Geophys. Res.-Oceans, 108, 3086, doi:10.1029/2002JC001321, 2003.

Takashima, T.: Polarization Effect On Radiative-transfer In Planetary Composite Atmospheres With Interacting Interface, Earth Moon Planets, 33, 59-97, doi:10.1007/BF00054709, 1985.

Varnai, T. and Marshak, A.: View angle dependence of cloud optical thicknesses retrieved by Moderate Resolution Imaging Spectroradiometer (MODIS), J. Geophys. Res.-Atmos., 112, D06203, doi:10.1029/2005JD006912, 2007.

von Schönermark, M., Geiger, B., and Röser, H.-P. (Eds.): Reflection Properties of Vegetation and Soil With a BRDF-Data base, vol. 1, Wissenschaft und Technik Verlag, Berlin, 2004.

Wendisch, M. and Yang, P.: Theory of Atmospheric Radiative Transfer A Comprehensive Introduction, Wiley-VCH Verlag GmbH \& Co. KGaA, Weinheim, Germany, ISBN: 978-3-52740836-8, 2012.

Wendisch, M., Müller, D., Schell, D., and Heintzenberg, J.: An airborne spectral albedometer with active horizontal stabilization, J. Atmos. Ocean. Technol., 18, 1856-1866, 2001.

Wendisch, M., Pilewskie, P., Jäkel, E., Schmidt, S., Pommier, J., Howard, S., Jonsson, H. H., Guan, H., Schröder, M., and Mayer, B.: Airborne measurements of areal spectral surface albedo over different sea and land surfaces, J. Geophys. Res., 109, D08203, doi:10.1029/2003JD004392, 2004. 